\title{
IdeAs
}

Idées d'Amériques

13 | 2019

La photographie documentaire contemporaine dans les Amériques

\section{« Había en su mirada más palabras que en cualquier voz » : Mineros, documentaire photographique et anthropologique des mines boliviennes}

"Había en su mirada más palabras que en cualquier voz": Mineros, documental fotográfico y antropológico de las minas bolivianas "Había en su mirada más palabras que en cualquier voz": Mineros, photographic and anthropologic documentary of Bolivian mines

\section{Baptiste Lavat}

\section{OpenEdition}

Journals

Édition électronique

URL : http://journals.openedition.org/ideas/4902

DOI : $10.4000 /$ ideas.4902

ISSN : 1950-5701

Éditeur

Institut des Amériques

Référence électronique

Baptiste Lavat, « « Había en su mirada más palabras que en cualquier voz »: Mineros, documentaire photographique et anthropologique des mines boliviennes », IdeAs [En ligne], 13 | 2019, mis en ligne le 01 mars 2019, consulté le 05 mai 2019. URL : http://journals.openedition.org/ideas/4902 ; DOI : 10.4000/ideas.4902

Ce document a été généré automatiquement le 5 mai 2019.

\section{c) $(\$)$}

IdeAs - Idées d'Amériques est mis à disposition selon les termes de la licence Creative Commons Attribution - Pas d'Utilisation Commerciale - Pas de Modification 4.0 International. 


\section{« Había en su mirada más palabras que en cualquier voz » : Mineros, documentaire photographique et anthropologique des mines boliviennes}

"Había en su mirada más palabras que en cualquier voz »: Mineros, documental fotográfico y antropológico de las minas bolivianas

"Había en su mirada más palabras que en cualquier voz »: Mineros, photographic and anthropologic documentary of Bolivian mines

Baptiste Lavat

\section{Rencontre avec la mine}

1 Tour à tour effrayés ou combattifs, interrogatifs ou hagards, résignés ou volontaires, les regards des mineurs immortalisés par les clichés du photographe Jean-Claude Wicky (1946-2016) dans son ouvrage Mineros. Bolivia (Wicky J.C., 2007) laissent difficilement indifférent. Fruit de dix-sept années de séjours réguliers dans les mines boliviennes, les soixante-treize photos en noir et blanc de ce livre, dont une majorité furent prises à l'intérieur des galeries minières, constituent à la fois un riche et poignant travail documentaire photographique, une œuvre d'art sobre, élégante et impressionnante, et un témoignage âpre mais respectueux des conditions de vie de milliers de boliviens s'engouffrant quotidiennement dans les profondeurs de la terre pour gagner leur vie.

D’ordinaire plutôt méfiants ou réservés à l'égard des étrangers prétendant entrer dans le monde sous-terrain à leurs côtés, les mineurs de la région de Potosi ont pourtant progressivement ouvert les portes de leur quotidien et accordé leur confiance au photographe suisse venu avec obstination les prendre en photo de longues années durant 
au plus profond de la mine. C'est ainsi qu'une véritable affection est née entre JeanClaude Wicky et ceux qui auraient au départ pu n'être que les sujets de ses photos, et dont il devint finalement l'ami et le collègue au fil de ses longs et nombreux séjours.

Figure 1 : Jean-Claude Wicky.

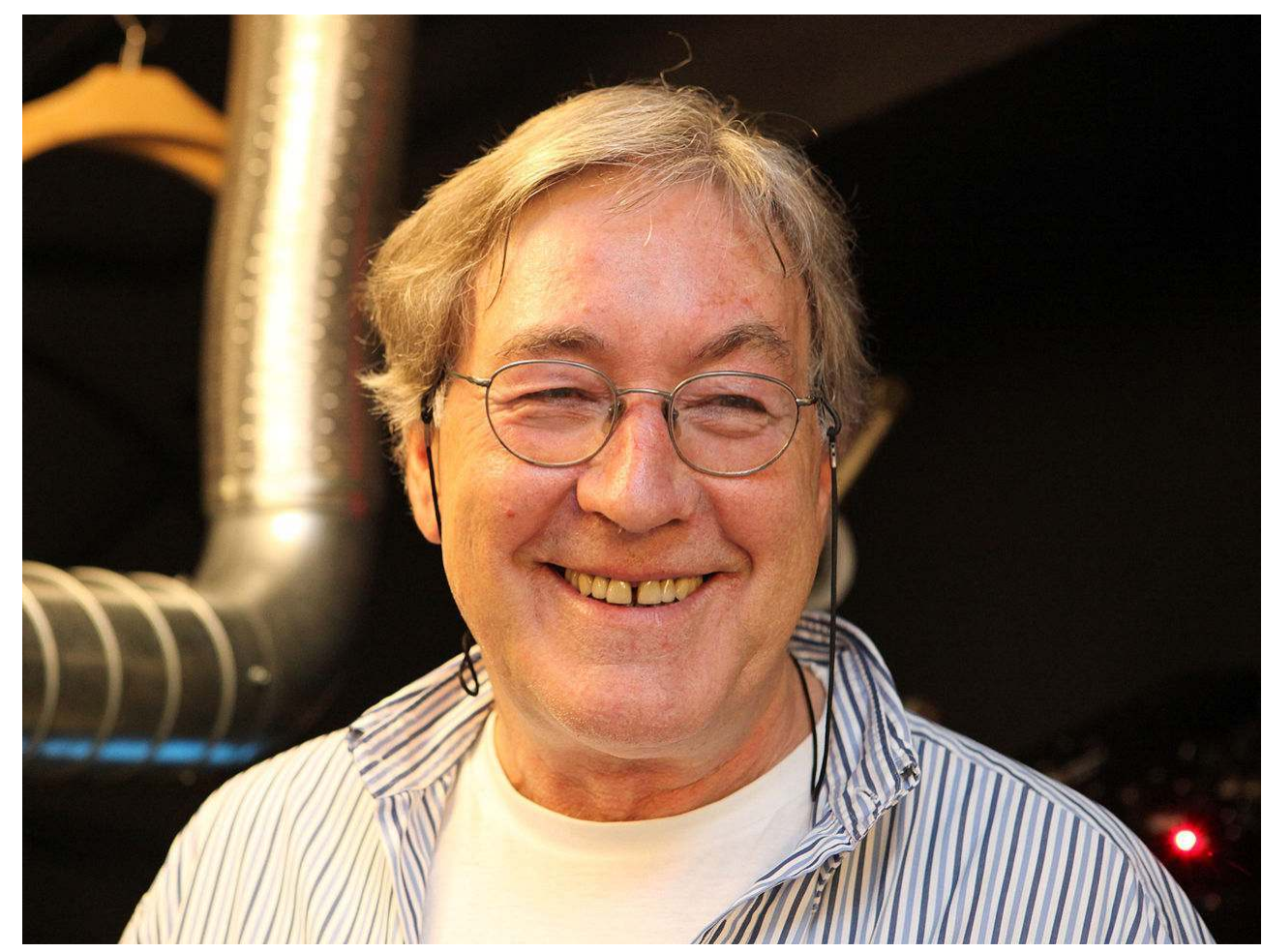

(c) Jan Boesch, 2010

Né en 1946 à Moutier, dans le canton suisse de Berne, si lointain tant géographiquement que culturellement des mines de Potosi, Jean-Claude Wicky eut un parcours pour le moins singulier. Après une formation à l'école de commerce de Bienne et trois années comme fonctionnaire des douanes, il devint footballeur en Ligue Nationale puis décida, en 1969, de faire le tour du monde dans un grand voyage de plusieurs années (1969-1975). C'est au Japon qu'il découvrit la photo, à laquelle il s'initia en autodidacte entre 1972 et 1973, avant de s'établir comme photographe professionnel au retour de son long périple en Asie, Australie et Amérique latine. Il ne cessa de parcourir le monde et découvrit ainsi la Bolivie en 1974, alors qu'elle était encore sous la coupe du gouvernement militaire d'Hugo Bánzer ${ }^{2}$ et participait activement à l'opération Condor. Elle sut néanmoins le séduire, en dépit de ce contexte peu hospitalier, de la dureté du climat et de l'altitude des HautsPlateaux ainsi que de son faible développement économique, qui en faisait à l'époque le pays le plus pauvre d'Amérique du Sud. Une dizaine d'années plus tard, elle deviendrait même la deuxième patrie de cœur de Wicky et le lieu emblématique de sa carrière artistique :

Quand je suis arrivé en Bolivie pour la première fois, j'ai découvert un pays différent des autres, peut-être plus authentique, peut-être plus mystérieux que les autres pays d'Amérique latine. J'adore ces paysages un peu arides où le temps feint de passer, ainsi que la tendresse et la gentillesse de ses gens. J'ai succombé au charme de la Bolivie. [...] Entre 1984 et 2001, j'y ai voyagé presque tous les ans pour explorer une trentaine de districts miniers ${ }^{3}$. 
La rencontre de Wicky avec la Bolivie et ses mineurs changea drastiquement et durablement le regard qu'il portait sur le monde : pendant près de deux décennies, il fut comme habité par ce pays et voyagea chaque année dans les Hauts-Plateaux de Potosi pour observer, comprendre, vivre et ressentir la mine jusque dans sa chair. Dans un entretien donné lors de la présentation de ses photos au musée de l'Elysée de Lausanne en 2002, le photographe raconte un rêve qu'il fit alors qu'il s'aventurait depuis plusieurs années déjà dans les entrailles de la terre aux côtés des mineurs : « Une nuit j'ai rêvé que j'étais dans une galerie où il faisait tellement chaud, je transpirais tellement que j'avais enlevé ma peau et l'avais mise à sécher sur un fil. Je me suis réveillé en sursaut parce que je ne savais plus comment la remettre. » (Wicky, J.C., 2007: 23) Un tel rêve, qui aurait certainement intéressé son compatriote le docteur Carl Gustav Jung, illustre bien la profonde identification de Wicky avec l'univers qu'il photographiait, et qui semble avoir marqué son inconscient au moins autant que sa conscience et sa production artistique.

Figure 2 : Pousser deux tonnes, Colquiri.

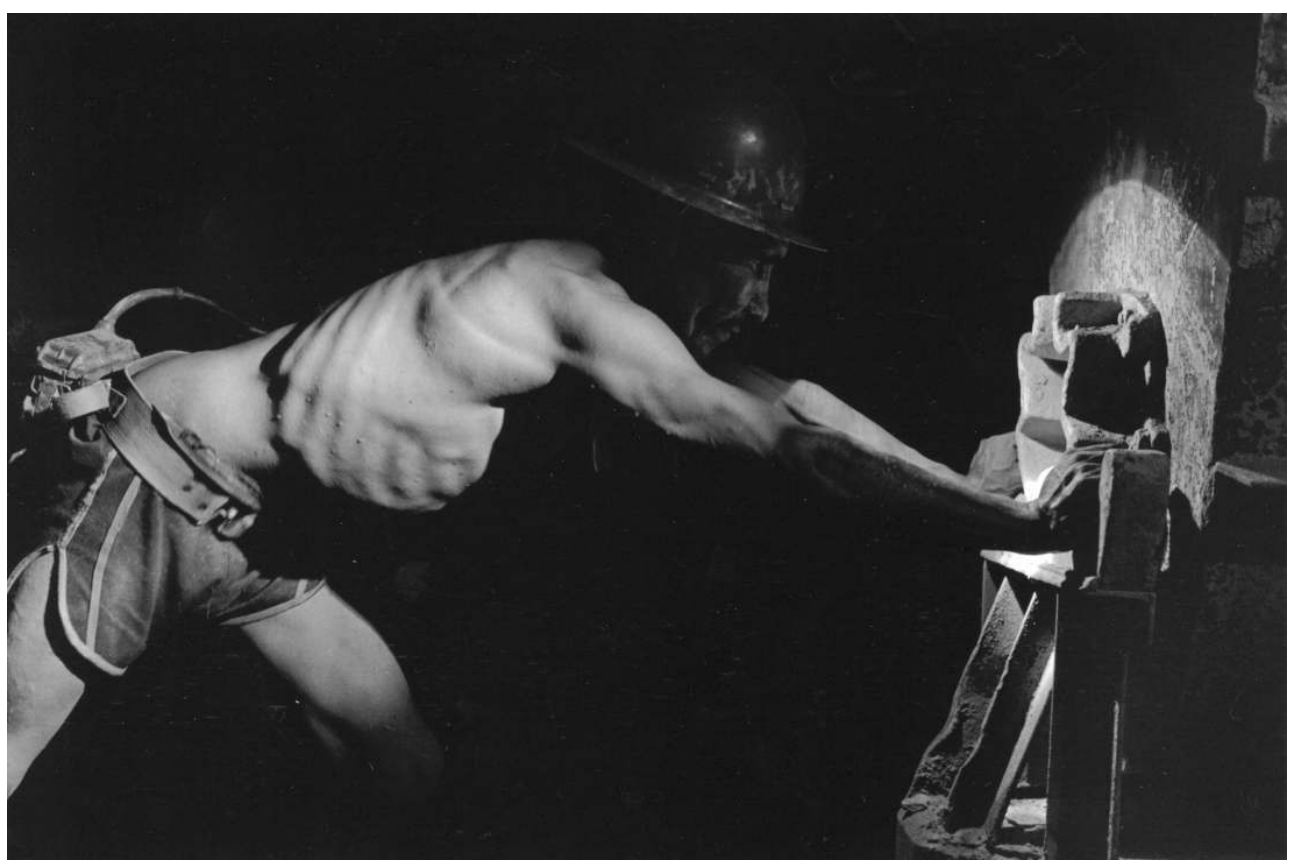

(c) Jean-Claude Wicky, 2002 (reproduit avec l'aimable autorisation de l'ayant-droit).

\section{Potosi, d'un siècle à l'autre}

5 Plus qu'un simple livre de photographies, Mineros. Bolivia est le témoignage de cette rencontre entre un photographe suisse, arrivé un peu par hasard en Amérique du Sud, et les régions andines de la Bolivie, pays qui fut des décennies durant le fournisseur de richesses minérales de l'Europe et forgea son économie sur le secteur minier, comme le rappelle fort bien Jean Pierre Lavaud :

La Bolivie [...] est économiquement un pays minier. Toute son histoire a été marquée par ce destin minier depuis l'extraordinaire épopée du Cerro Rico de Potosi qui a fait du Haut-Pérou (puis de la Bolivie), le principal producteur d'argent pendant trois siècles, et qui a forgé la légende de sa richesse, jusqu'à l'exploitation du filon stannifère ; destin paradoxal d'un pays dont les trésors paraissent échapper 
à ses habitants, voué à une pauvreté répétitive et comme épuisé d'avoir été tant creusé. (Lavaud, J.-P., 1991 : 145-171)

La ville de Potosi, grâce à son fameux Cerro Rico, fut effectivement une importante cité à l'époque coloniale, sacrée "ville impériale » par l'empereur Charles Quint en 1573 et peuplée de plus de 120000 âmes $^{4}$ à la fin du XVI ${ }^{\mathrm{e}}$ siècle. Considérée comme ayant fourni près du tiers de la production globale d'argent du continent à l'époque coloniale, et plus de soixante pour cent durant le Xvi siècle (Helmer, M., 1951 : 21), elle devint l'un des joyaux du vice-royaume du fait de son économie fleurissante, dont témoigne aujourd'hui encore son riche patrimoine architectural constitué d'églises, de théâtres et d'imposants édifices coloniaux tels que l'ancienne Maison royale de la monnaie, fondée en 1572 pour se substituer à celle de Lima.

Figure 3 : Descripción del Cerro Rico e Imperial Villa de Potosí (détail), Gaspar Miguel de Berrío, 1758, Musée colonial Charcas.

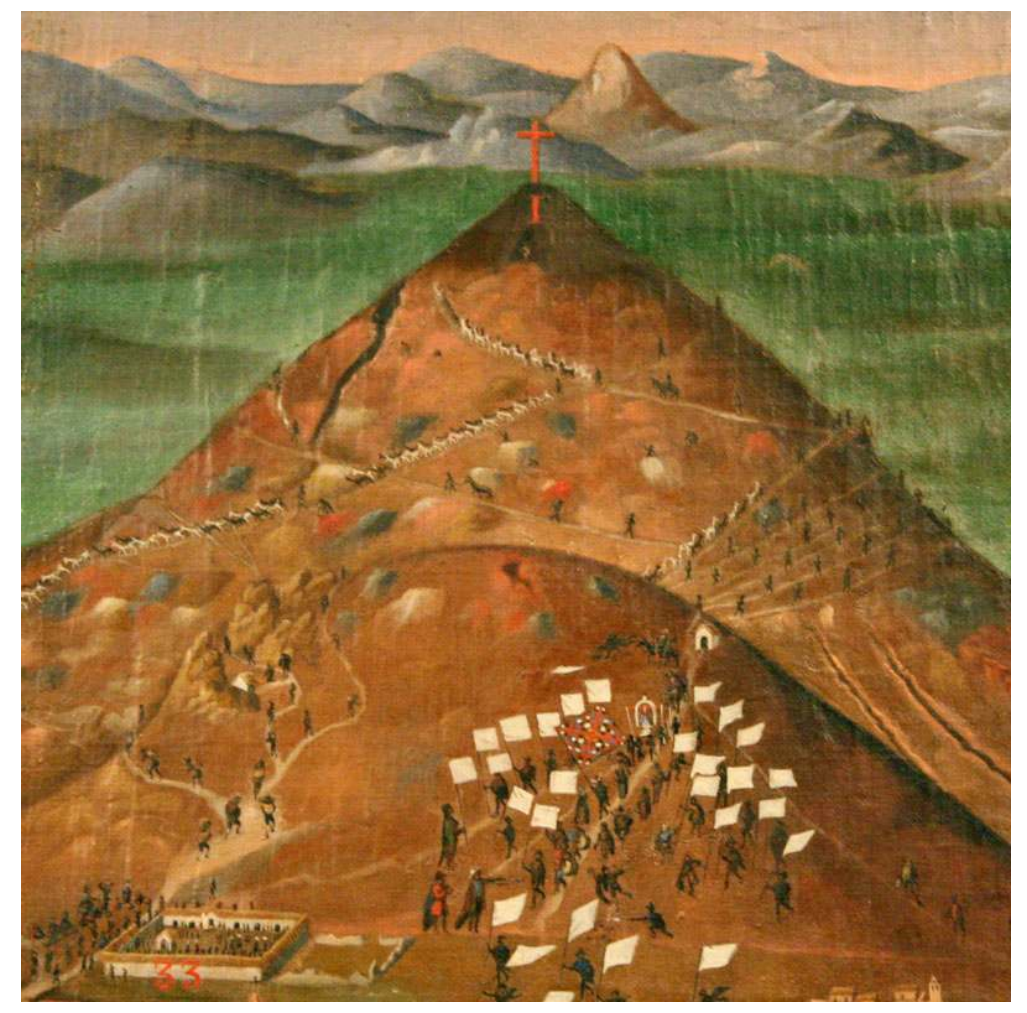

7 Autant de vestiges de la vitalité artistique, culturelle et économique d'antan, qui contrastent brutalement avec le statut actuel de capitale du département le plus pauvre $\mathrm{du}$ pays. Les deux époques semblent ainsi se confondre ou se superposer, comme le suggère Wicky, se remémorant certains de ses échanges avec les mineurs :

Quand ils me racontaient ce que leur avait dit cette terre, leurs voix semblaient venir du passé, comme si l'histoire récente se confondait avec l'histoire de la Colonie, et que rien n'avait changé. Je trouvais souvent qu'il y avait dans leur regard plus de mots que dans quelque voix que ce soit. (Wicky J.-C., 2010)

Cette évocation de l'histoire coloniale par Wicky convoque instantanément l'imaginaire de l'exploitation dont les populations indigènes firent l'objet, réduites en esclavage par les colons qui récupérèrent à leur avantage le travail d'extraction de la mita de l'époque inca. Ce faisant, Wicky fait dialoguer des époques bien distantes mais partageant néanmoins cette dimension d'asservissement d'une main d'œuvre dépossédée des 
richesses qu'elle exploite, dans une rencontre atemporelle déjà suggérée par Jean-Pierre Lavaud:

L'histoire récente de la mine est ancrée dans une histoire ancienne qui se perd dans la mythologie "enchantée" des origines. L'extraction intensive des minerais d'argent et l'asservissement des indigènes contraints à la mita remontent aux débuts de la colonisation; ils se confondent avec elle. Et les dirigeants syndicalistes actuels ne manquent pas de rappeler souvent que les profits de l'exploitation minière sont toujours allés à des étrangers, colonisateurs, magnats de l'étain expatriés, acheteurs impérialistes; plus exactement, ils insistent sur le fait que la richesse locale n'a que rarement profité aux mineurs eux-mêmes, aliénés du produit de leur travail. (Lavaud, J.-P., 1991 : 145-171)

Après une époque de flambée du prix de l'étain (Bernard, J.-P., 1967), qui vit l'économie de la région et du pays s'envoler, bien que ses principaux bénéficiaires fussent un groupe réduit d'oligarques connus sous le nom de «barons de l'étain ${ }^{5}$ », la Révolution de 1952 nationalisa les mines et créa la puissante Corporación Minera de Bolivia (COMIBOL), qui administra alors les mines du pays. Sa gestion connut cependant un certain nombre de limites et d'erreurs, et les conditions de travail et de vie de l'immense majorité des mineurs ne s'améliorèrent que très peu, comme en témoignèrent les grandes révoltes de 1967 et 1980, pour ne citer que deux des massacres liés à la répression des mouvements de protestation des mineurs par le gouvernement bolivien au cours du xxe siècle (Dunkerley, J., 2003) ${ }^{6}$. Le « massacre de la San Juan » (1967), porté au cinéma par le réalisateur bolivien Jorge Sanjinés dans le film El coraje del pueblo ${ }^{7}$, fit aussi l'objet d'un hommage par l'auteur uruguayen Eduardo Galeano, publié dans le dernier volume de sa trilogie Memoria del fuego , sous le titre « La fiesta de San Juan »:

En esta noche de San Juan, mientras ocurre la mejor de las fiestas, el ejército se agazapa en las montañas. Casi nada se sabe aquí de los guerrilleros del lejano río Ñancaguazú, aunque dicen que pelean por una revolución bella y jamás vista, como la mar, pero el general Barrientos cree que en cada minero anida un taimado terrorista. Antes del amanecer, al final de la fiesta de San Juan, un huracán de balas arrasa el pueblo de Llallagua. Parece fulgor de huesos la luz del nuevo día. Después el sol se esconde tras las nubes, mientras los parias de la tierra cuentan sus muertos y en carretillas los llevan. (Galeano, E., 1986) 


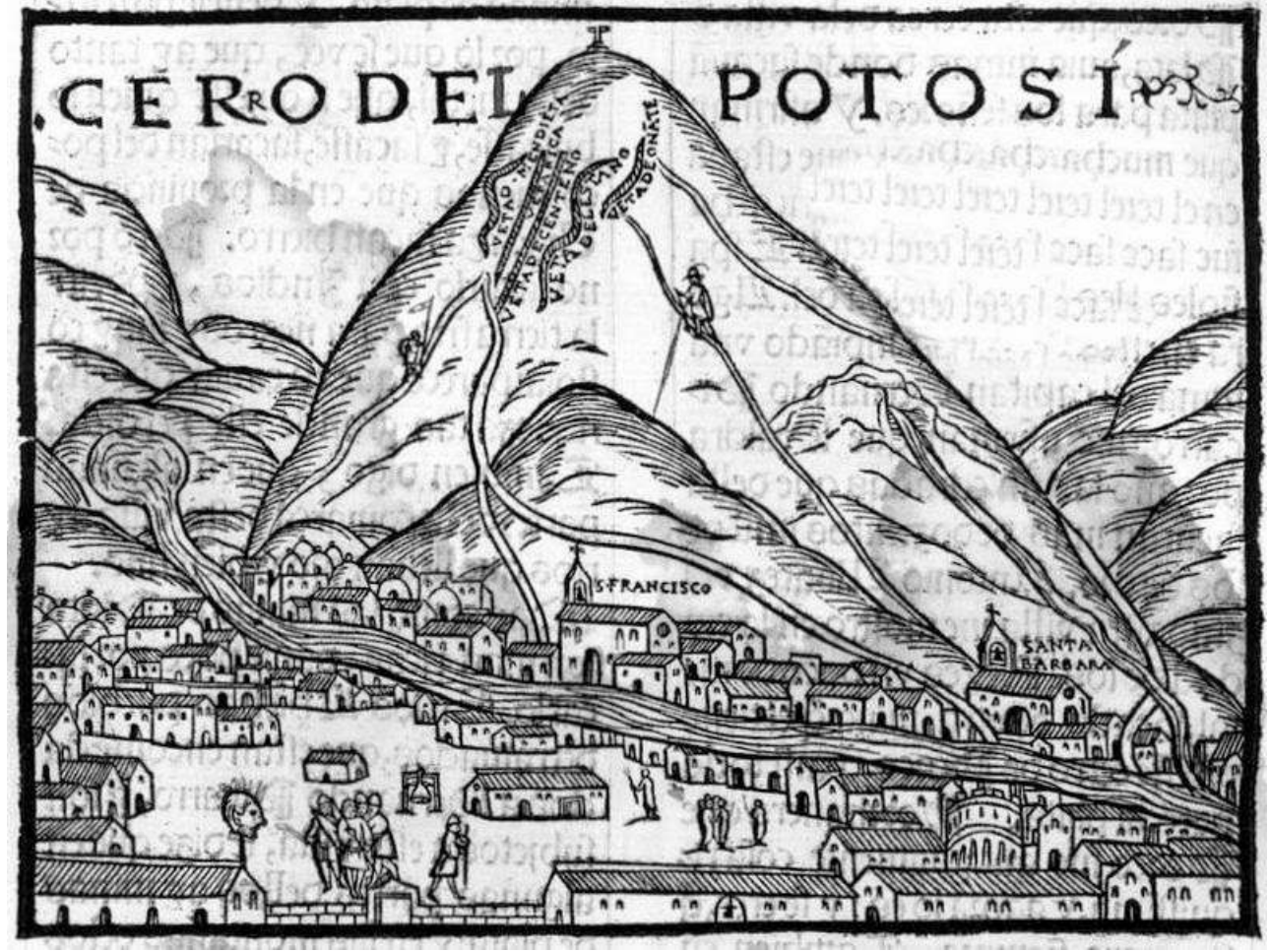

Outre les différents épisodes de répression des mineurs, la chute du cours des métaux lors de la grande crise de 1985 et la campagne de privatisation qui s'en suivit, avec l'application des réformes néolibérales imposées à l'ensemble du sous-continent, eurent indéniablement de terribles conséquences sur le secteur minier bolivien. Près de 27000 mineurs de la Corporación Minera de Bolivia furent licenciés en août 1986 (Décret suprême nº 861, 1 mai 2011), déclenchant un important mouvement de protestation le 21 du même mois avec la «Marcha por la vida» de plusieurs milliers de personnes entre les villes d'Oruro et de La Paz. La marche n'en fut pas moins un échec, puisqu'elle fut dissoute une semaine plus tard avant de parvenir à son objectif, le gouvernement de Paz Estenssoro ayant déclaré l'état de siège et déployé l'armée à quelques dizaines de kilomètres de La Paz. Suite à cet épisode, la situation des mineurs de la COMIBOL changea drastiquement, ainsi que celle de l'ensemble du secteur minier national :

Le secteur minier se retrouve divisé en trois branches : un secteur étatique quasi à l'abandon, un secteur privé qui récupère la gestion des principaux gisements et un secteur de coopératives minières qui se construit par les travailleurs eux-mêmes dans les mines marginales du pays, sans ressources économiques ni capacités d'investissement. Ainsi s'élabore une industrie minière dualiste entre un secteur privé de grande productivité et un secteur de coopératives rudimentaires. (Le Gouill, C., 2016 : 49-69)

11 Face à la crise, l'État dut en effet privatiser une grande partie des mines nationalisées en 1952 ou les donner en location à des coopératives qui employèrent alors une part croissante des travailleurs, dans des conditions souvent très précaires. Cette dure réalité semble avoir eu son importance dans la démarche du photographe suisse, qui rappelle volontiers combien les ressources de la région «feront la fortune de quelques-uns et la misère du pays » (Wicky J.C, 2010), emboîtant ainsi le pas au mineur Paulino Calisaya qui 
lui avait déclaré que «la richesse de notre sous-sol a toujours été la source de notre pauvreté » (ibid.).

Loin de se contenter de présenter des portraits et clichés de la vie quotidienne des mineurs avec un regard extérieur, Wicky fit le choix de vivre à leurs côtés, dans des conditions particulièrement difficiles pour une personne non habituée à la mine, afin de proposer un travail et une réflexion se voulant le plus objectifs possible sur les conditions de vie de ceux dont il considère qu'ils ont certainement « le métier le plus dur du monde » (Wicky, J.-C., in Zig Zag Café, 2003). C'est probablement pourquoi son ouvrage Mineros. Bolivia ne saurait être réduit à la dimension artistique et technique des photographies proposées, car il ne fut possible que grâce à un long processus d'apprivoisement mutuel, à la construction d'une relation de confiance et de respect qui firent progressivement du rapport entre le photographe et ses sujets une histoire d'amitié, comme l'affirme Wicky lui-même dans son documentaire Todos los días la noche :

Dans mon laboratoire, chaque photographie qui apparaît au révélateur me rappelle une histoire : de solidarité, de tendresse, d'amitié, de générosité, d'hospitalité. [...] J'ai durant de longs mois partagé la vie des mineurs, leurs joies et leurs souffrances, leur révolte et leurs terribles eaux-de-vie. En réalisant ce travail, mon objectif n'était pas de les photographier avec des lentilles faisant voir la vie en rose, ni de faire du sensationnalisme misérabiliste. J'ai tenté de raconter la vie de ces gens de la mine avec respect. [...] Ce travail est mon chant d'amitié au peuple bolivien et à tous ceux dont le labeur quotidien consiste à chercher leur destin dans les profondeurs de la terre. (Wicky J.C., 2007: 26)

Figure 5 : Elías Colque, Viloco.

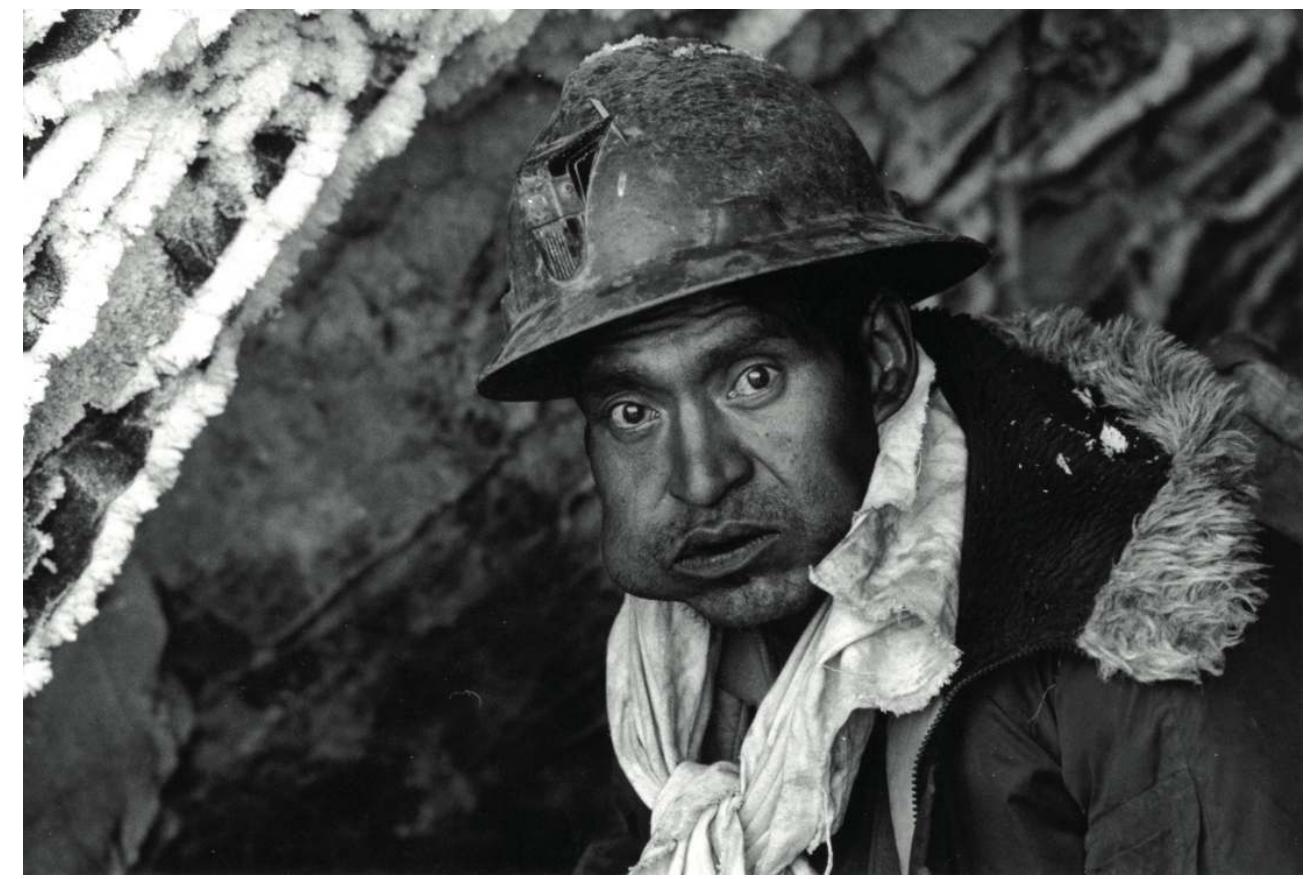

(c) Jean-Claude Wicky, 2002 (reproduit avec l'aimable autorisation de l'ayant-droit).

\section{Une anthropologie en photos}

C'est en partageant leur quotidien avec les mineurs que Wicky put véritablement pénétrer dans leur univers et en découvrir personnellement les conditions de travail: 
manque d'oxygène lié à l'altitude, grandes variations de température, menaces permanentes d'un éboulement ou d'une poche de dioxyde de carbone, infrastructures obsolètes, etc. Cependant, il découvrit aussi les relations de fraternité et d'entraide entre les mineurs, qui l'accueillirent progressivement jusqu'à le considérer comme l'un des leurs, constatant qu'il n'hésitait pas à s'aventurer avec eux dans certaines des galeries les plus reculées et périlleuses.

14 C'est donc aussi contre l'image réductrice d'une Bolivie indigente que souhaitait lutter le photographe, déplorant que le pays ne soit souvent connu à l'étranger qu'en raison des nombreux coups d'État qui ont marqué sa vie politique, du trafic de cocaïne dont il est devenu l'un des rouages en tant qu'important producteur de feuille de coca, ou plus récemment de la présidence d'Evo Morales (depuis 2006), lui-même ancien syndicaliste cocalero.

Figure 6 : Sans titre, Potosi.

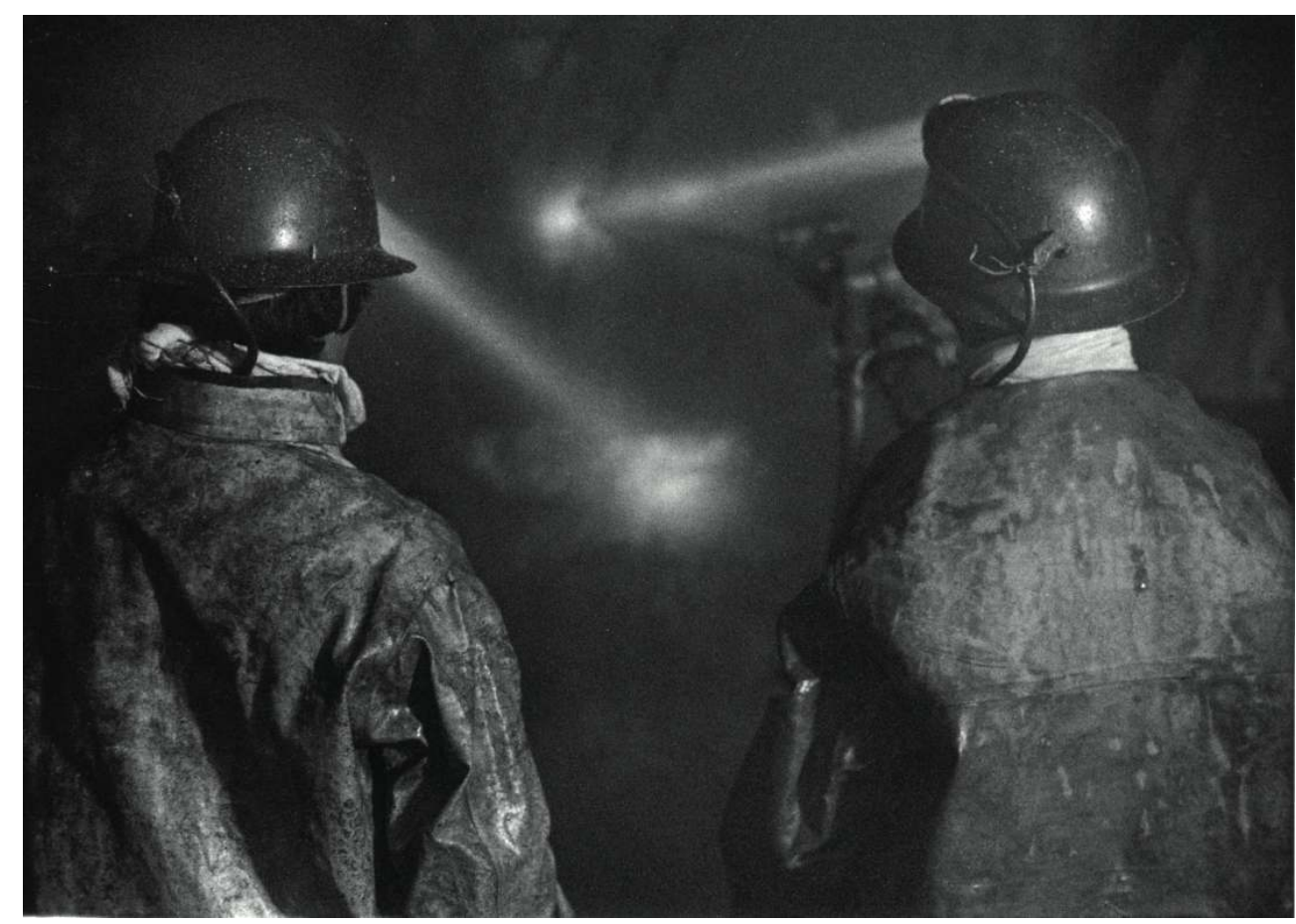

(c) Jean-Claude Wicky, 2002 (reproduit avec l'aimable autorisation de l'ayant-droit).

15 Si le portrait que dresse Jean-Claude Wicky des conditions de l'activité extractive dans les coopératives boliviennes est sans concession, celui des mineurs est au contraire empli de l'admiration de celui qui se disait touché autant qu'impressionné par la force de caractère et la résilience de ceux qu'il a accompagnés et photographiés dix-sept années durant :

Leur solitude me toucha; une solitude de plusieurs siècles. [...] Mon intention était de raconter la vie de ces mineurs, leur sacrifice, leur dignité. Quand ils réalisèrent que jour après jour je m'enterrais à leurs côtés dans les ténèbres de ce labyrinthe dantesque où ils défient la roche et échangent avec le diable, je fus reconnu comme l'un des leurs, travaillant simplement avec des outils différents. (Ibid.)

Ces «outils différents» - un appareil photo et un éclairage portatif sommaire - lui permirent de reproduire aussi fidèlement que possible l'obscurité des galeries où il prit ses clichés, même si le défi constant des conditions exceptionnelles qu'elles présentaient 
lui posa bien des soucis, sans compter qu'il lui semblait particulièrement difficile de restituer en photo la réalité du monde dans lequel évoluent les mineurs :

Je ne m'étais pas rendu compte de l'ampleur de la tâche. Comment photographier l'humidité, la chaleur, le manque d'oxygène, l'odeur âcre du minerai qui imprègne les corps? Comment photographier l'obscurité de la mine, épaisse, plus impénétrable que la roche, qui fait disparaître tout sens de l'orientation, toute notion du temps et de la distance, l'obscurité qui te brûle les yeux et fait disparaître ton corps? (Ibid.)

S'il fut souvent amené à ressortir des souterrains sans même avoir pu prendre de photographies tant la tâche s'avérait difficile ou, dans certains cas, parce qu'il ne jugea pas opportun de le faire par égard pour ses camarades, Wicky réussit cependant à dresser un portrait visuel saisissant d'un monde présenté sans fard, sobre et parfois glaçant, brut et minéral tant dans ses sujets que dans sa texture. En dépit de conditions techniques et matérielles rendant très difficiles les prises de vue et le travail de la lumière, il sut retranscrire de façon très efficace certaines des caractéristiques "physiques" du lieu au spectateur.

Jouant sur le flou, le manque de lumière, la "matière" dans l'image, ou encore sur des cadrages très serrés nous plongeant dans l'étroitesse des galeries et permettant de saisir la sueur perlant sur les corps et la poussière qui s'y dépose, certains clichés retranscrivent avec force l'ambiance moite et poussiéreuse des galeries, jusqu'à faire parfois ressentir au spectateur un réel malaise doublé d'une étrange sensation d'enfermement.

Figure 7 : Sans titre, Potosi.

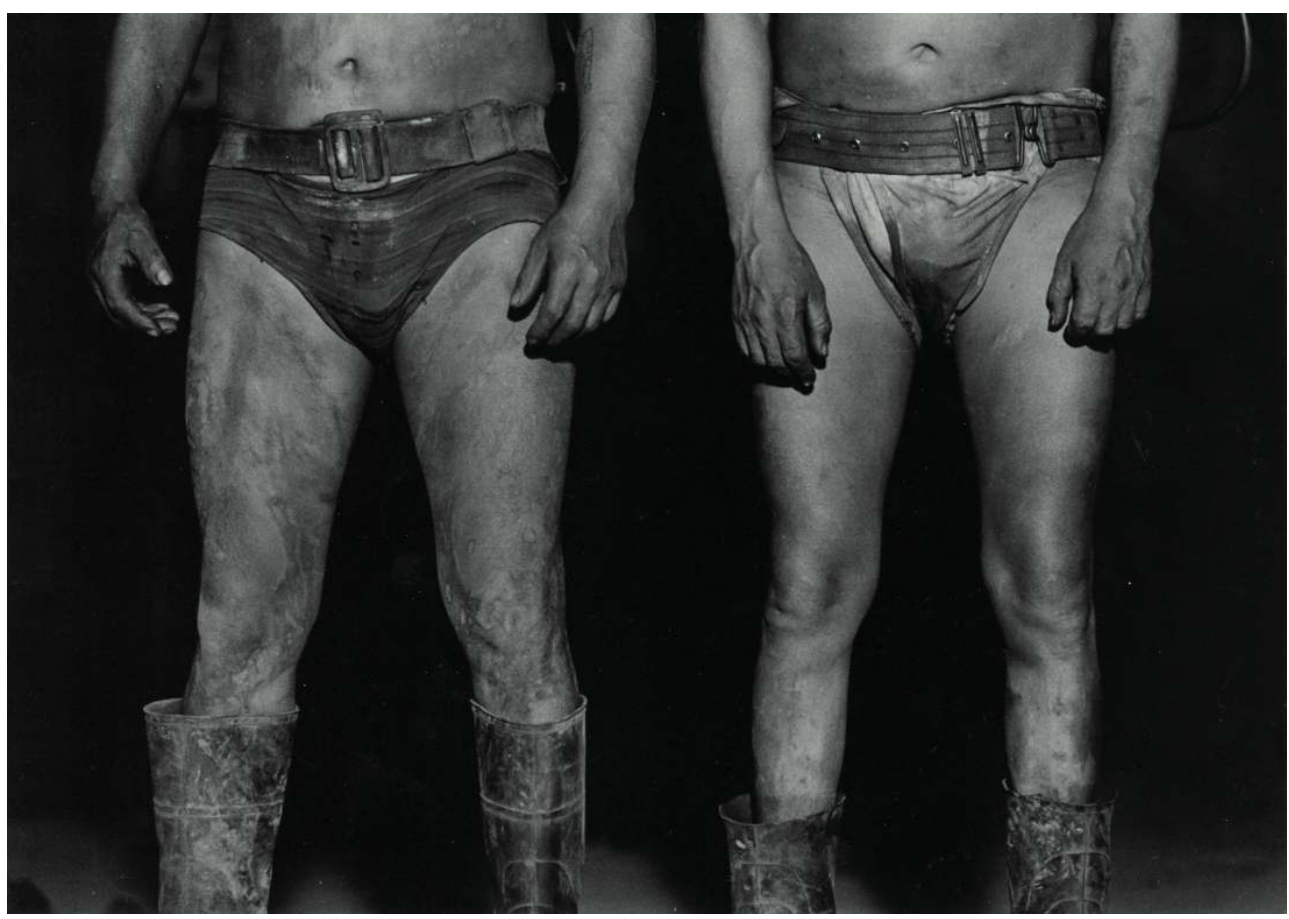

(c) Jean-Claude Wicky, 2002 (reproduit avec l'aimable autorisation de l'ayant-droit).

19 D'autres photographies, qui présentent en très gros plan certains des outils de travail de la mine ${ }^{8}$ ou la figure tutélaire des lieux nommée «Tío de la Mina ${ }^{9}$ » et les offrandes qui lui sont consacrées, cherchent à restituer non seulement l'atmosphère du lieu, mais aussi le 
microcosme qui s'y installe avec l'activité des mineurs, sortes de fourmis ouvrières enfouies dans un réseau dense et étroit de galeries constituant un royaume dont elles n'ont pas les clés et semblent moins les bénéficiaires que les victimes, à en croire le mineur Emilio Mena qui confia à Jean-Claude Wicky que «la mine me nourrit et me permet de vivre, mais elle me tue lentement » (Wicky J.C, 2010).

Figure 8 : Valentín Mamani, Potosi.

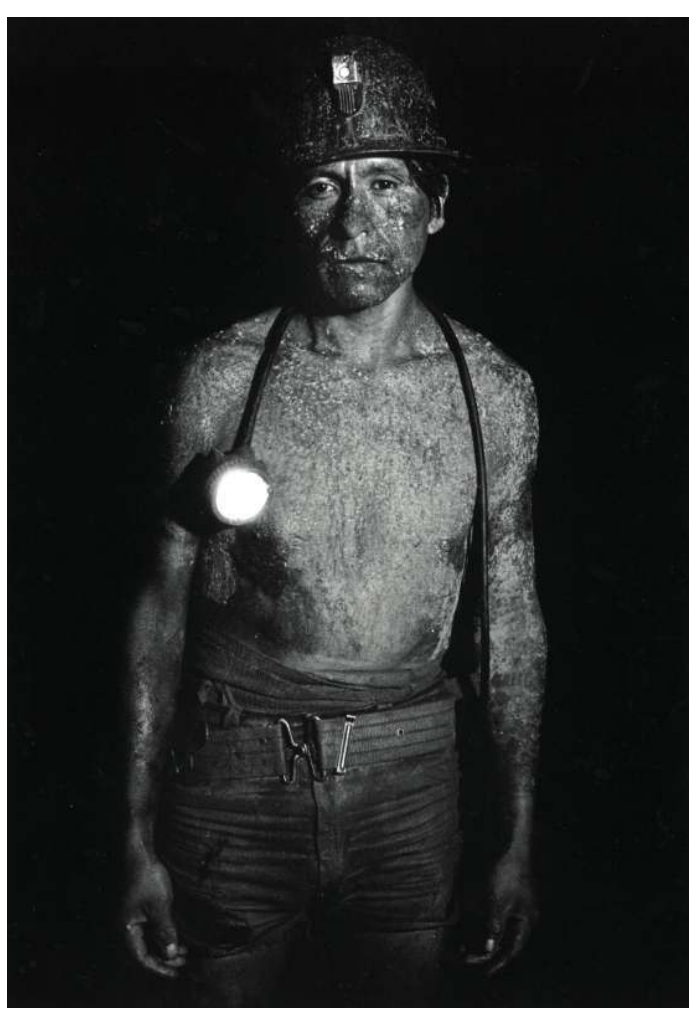

(c) Jean-Claude Wicky, 2002 (reproduit avec l'aimable autorisation de l'ayant-droit). 
Figure 9 : El Tío (le diable), Potosi.

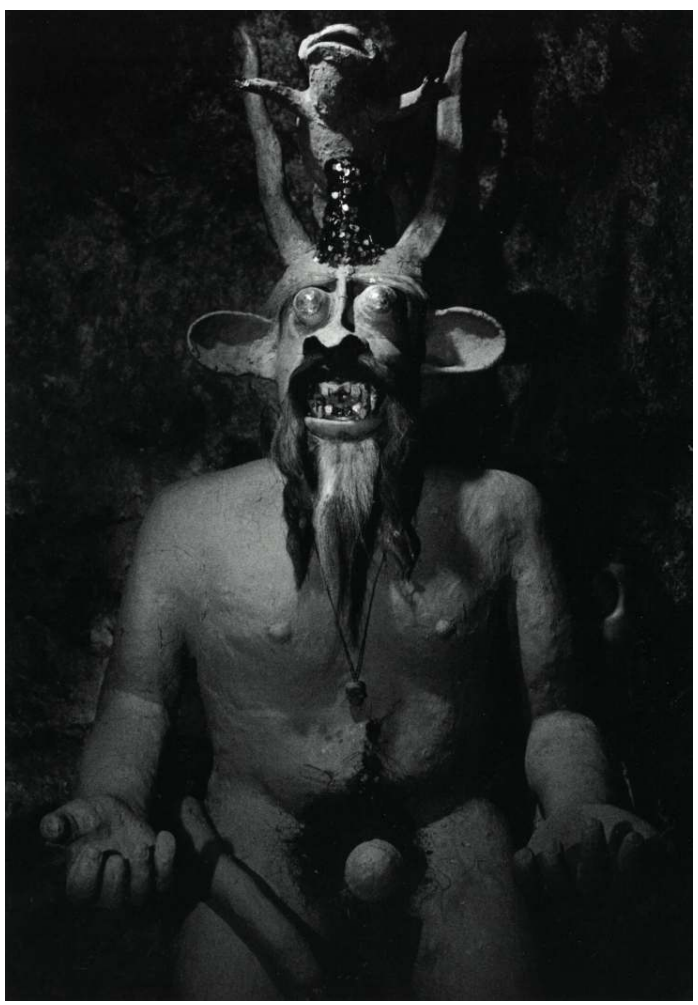

(c) Jean-Claude Wicky, 2002 (reproduit avec l'aimable autorisation de l'ayant-droit).

\section{Le diable ne rêve qu'en noir et blanc}

«Le diable ne rêve qu'en noir et blanc » (Rico, L., 2016), titre d'une chanson composée par le musicien et chanteur bolivien Luis Rico en hommage à son ami Jean-Claude Wicky, illustre poétiquement le parti-pris du photographe de proposer des clichés exclusivement en noir et blanc, choix qui semble presque évident pour dépeindre un monde dans lequel les couleurs sont altérées voire littéralement éclipsées par le manque de luminosité. Effectivement, la profondeur du noir occupe finalement la majorité de l'espace dans la plupart des photographies, devenant l'illustration même de l'obscurité dans laquelle évoluent les mineurs durant leurs journées de travail. En renforçant les ombres et les contrastes des corps et des visages, il donne parfois l'impression que certains sortent tout droit du néant, suspendus dans une sorte de vide qui souligne la solitude de ces hommes lorsqu'ils pénètrent sous terre. Car dans la mine, le temps semble comme en suspension et la vie plongée dans un rythme et une obscurité qui altèrent les sens et font perdre toute notion spatiotemporelle, comme en témoignait déjà l'écrivain bolivien René Poppe dans son journal Interior Mina : « La montre n'existe pas pour le mineur. [...] À l'intérieur de la mine, il mastique la coca, et quand elle devient aigre en bouche, il sait combien de temps est passé. » (Poppe R., 2003 [1986]: 40) ${ }^{10}$.

21 Les portraits de mineurs posant pour l'objectif plutôt que saisis spontanément dans leur effort jouent particulièrement sur cette utilisation d'un noir si dense qu'il en deviendrait presque oppressant, contribuant à faire se détacher plus encore les regards des fonds uniformément sombres, les lampes des casques allumées semblant constituer la seule 
source de lumière du cliché. Le jeu d'ombres qui en résulte renforce ainsi la profondeur de l'expression de visages certes différents mais tous extrêmement graves et donnant la sensation qu'une sorte de menace plane dans l'air, tapie dans la pénombre et l'immensité d'un silence à peine troublé par les coups de burin et les détonations de dynamite résonnant par intermittence dans les entrailles de la terre.

Figure 10 : Sans titre, Canyon d'Antequera.

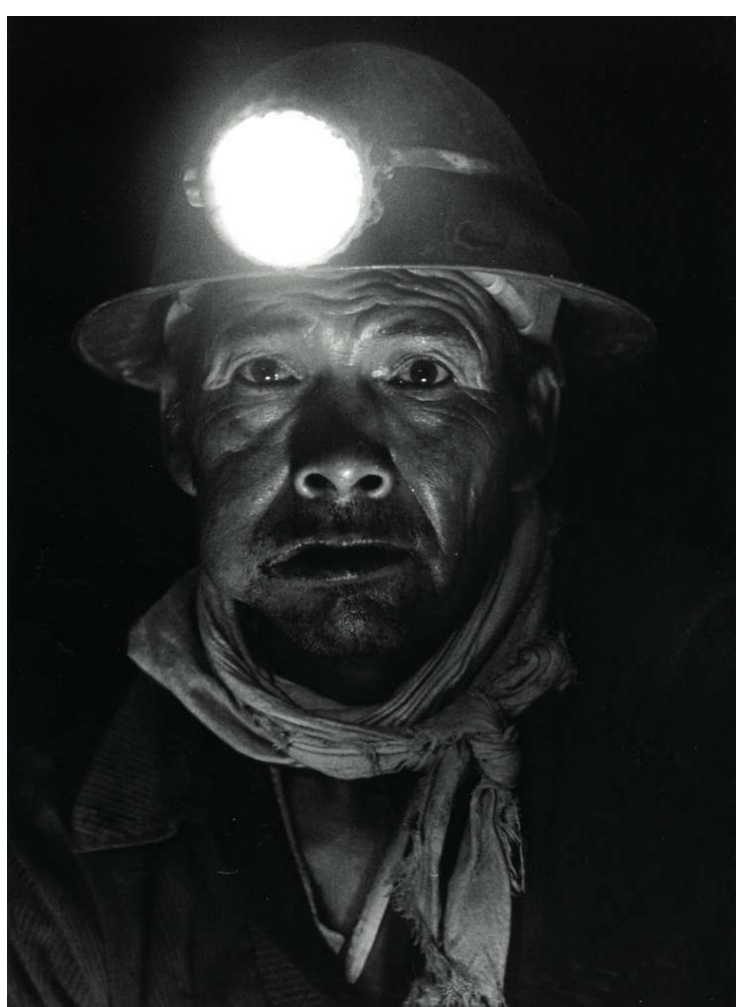

(c) Jean-Claude Wicky, 2002 (reproduit avec l'aimable autorisation de l'ayant-droit). 
Figure 11 : Sans titre, Chorolque.

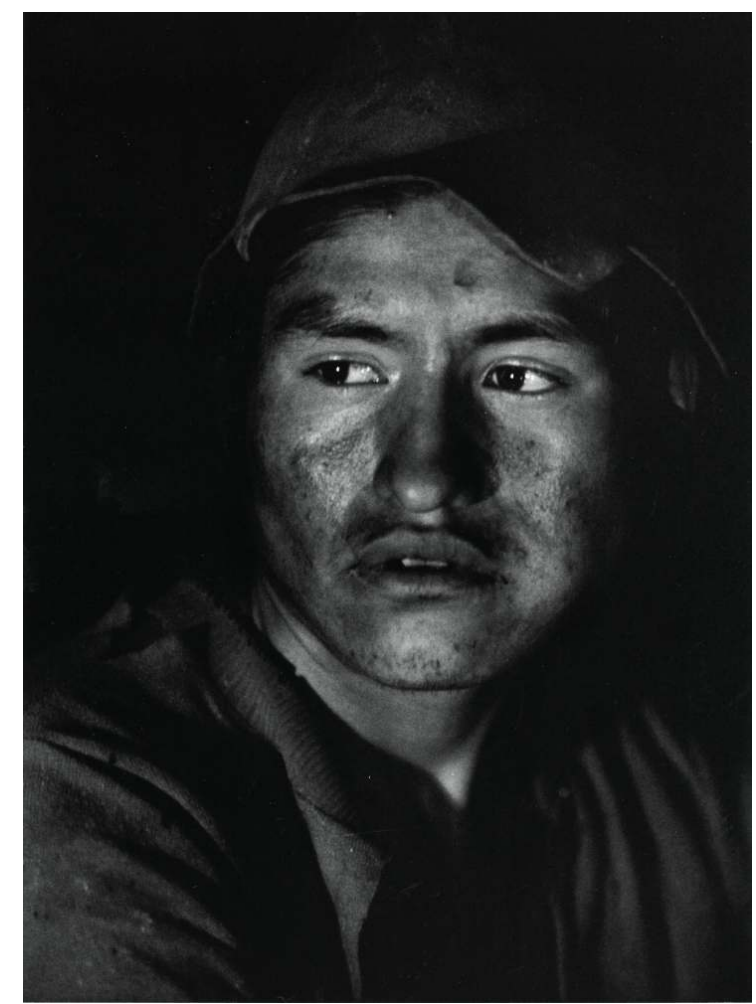

(c) Jean-Claude Wicky, 2002 (reproduit avec l'aimable autorisation de l'ayant-droit).

22 Un détail frappe rapidement le spectateur : pas une femme n'apparaît sur les clichés pris à l'intérieur de la mine. Et pour cause, leur présence y est interdite car elle pourrait importuner voire fâcher le Tío qui ne les tolère pas en son domaine, selon une croyance très ancrée en Bolivie ${ }^{11}$. Elles ne sont pas pour autant absentes de cet univers, se voyant généralement affectées au travail de palliri ${ }^{12}$, qui consiste à trier le minerai extrait du sous-sol pour en dégager les plus riches exemplaires, dont la concentration semble suffisante pour qu'ils soient raffinés par la suite. Un labeur qui, s'il présente l'avantage de ne pas se réaliser sous terre, reste extrêmement pénible, et pour lequel les palliris sont parfois secondées par leurs enfants, surtout lorsqu'elles sont veuves ou que leur mari n'est plus en condition de travailler sous terre pour des raisons de santé : la silicose, affection aiguë des poumons, touche nombre de mineurs, souvent tenus d'arrêter leur activité. 
Figure 12 : Deux palliris sur le chemin du travail, cordillère Las Tres Cruces.

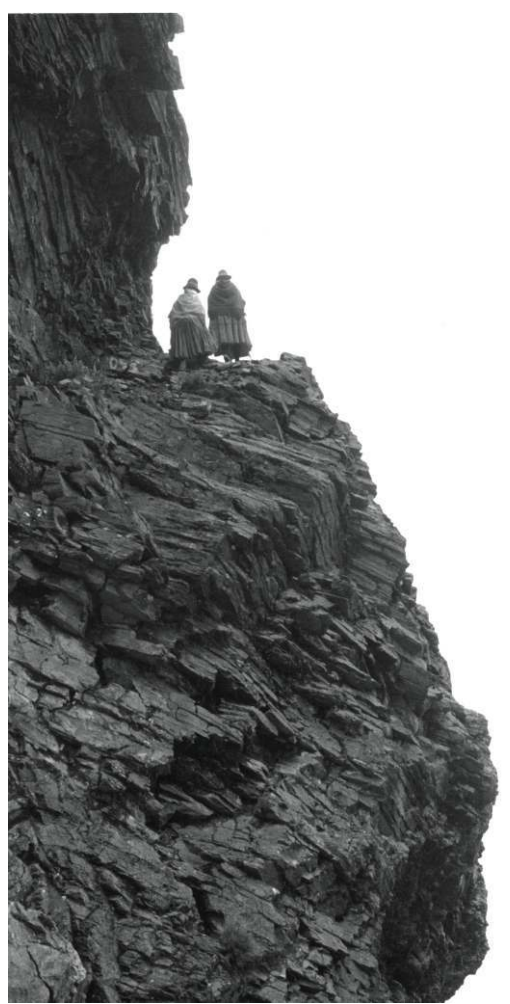

(c) Jean-Claude Wicky, 2002 (reproduit avec l'aimable autorisation de l'ayant-droit).

Après la plongée dans les galeries de la première partie de son ouvrage intitulée «Interior Mina », Jean-Claude Wicky choisit de proposer des photographies provenant non pas des profondeurs, mais de ce que les mineurs appellent communément «le campement » et qui désigne, dans le cas des régions photographiées, les baraques de fortune aménagées aux pieds de la montagne. Dans cette deuxième partie intitulée "Campamento ", vingthuit clichés proposent un parcours à l'extérieur de la mine, entre les baraquements des familles de mineurs, l'entrée des souterrains où sont réalisés les rituels propitiatoires et protecteurs, ou encore les flancs de montagne où s'accumulent les roches triées par les palliris.

Les photographies n'en sont pas moins poignantes, mais contrastent néanmoins fortement avec celles du chapitre "Interior Mina», ne serait-ce que par la présence de visages d'enfants et de quelques sourires qui détonnent avec l'âpreté d'un monde jusqu'alors perçu comme presque exclusivement grave et angoissant. Si quelques clichés dépeignent un quotidien qui semble connaître aussi ses moments de communion autour d'un match de football improvisé, de repas communautaires ou de fêtes liées au calendrier rituel andin, le panorama reste extrêmement austère : lits de camps rouillés, précarité des installations et visages burinés par le soleil de l'Altiplano et la rudesse du travail, que les sourires des enfants ne sauraient faire oublier. Mais une fois encore, l'agencement des clichés, leur qualité et leur sobriété évitent de tomber dans le piège d'une peinture larmoyante ou catastrophiste, et dressent simplement le constat d'une réalité dont le photographe parle d'ailleurs avec un respect, une admiration voire une tendresse qui ne font pas mentir ses photographies. Elles témoignent ainsi du fait qu'une vie, si rude soit-elle, existe là-haut, silencieuse et presque invisible, perdue dans les cimes 
de l'Altiplano. C'est là l'hommage de Wicky à ceux qui lui ont permis d'« entrer dans le pays, sans s'en rendre compte, par sa porte la plus douloureuse » (Wicky J.C., 2010).

Figure 13 : Mate de coca, Potosi.

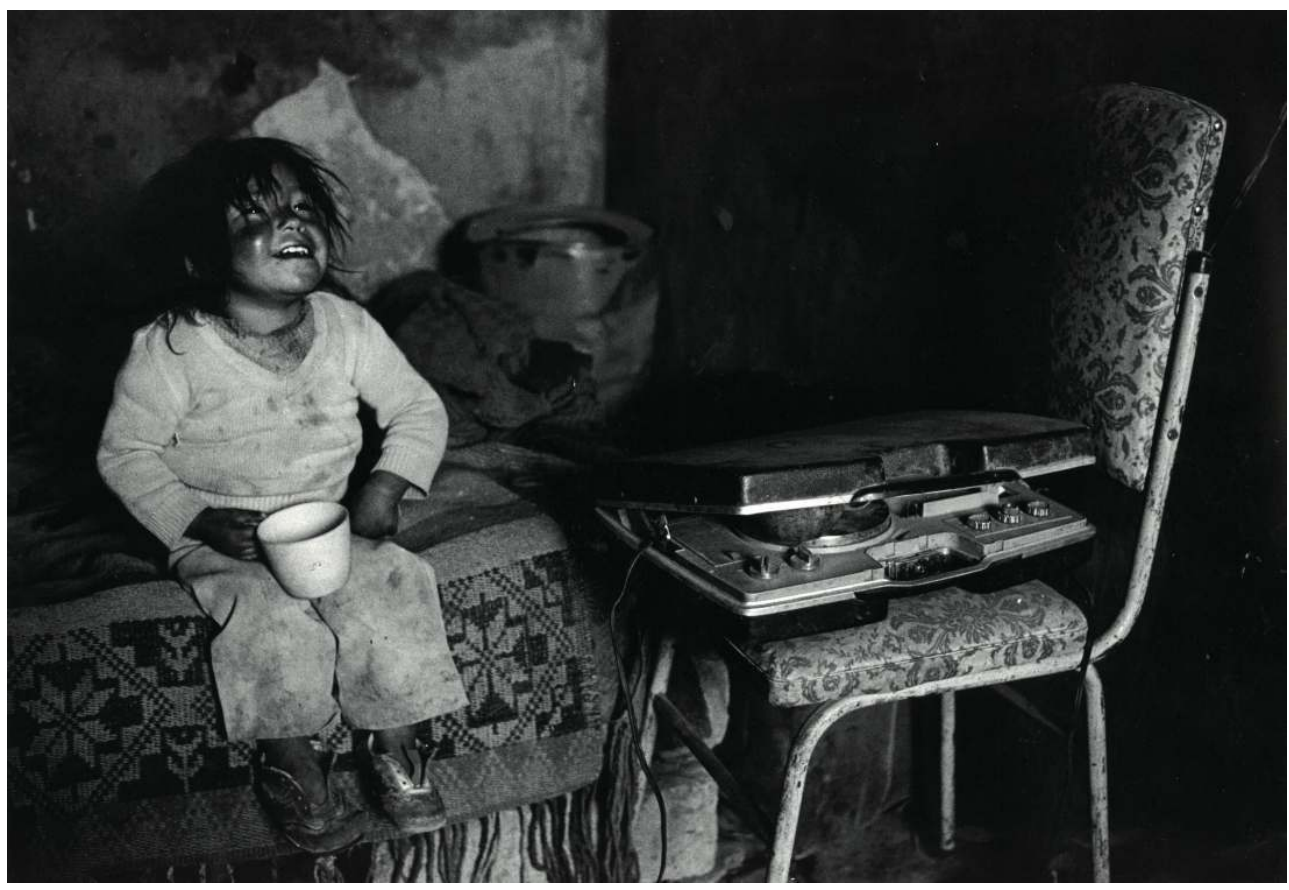

(c) Jean-Claude Wicky, 2002 (reproduit avec l'aimable autorisation de l'ayant-droit).

\section{Tous les jours, la nuit}

Ce n'est qu'en 1998, près de quinze ans après avoir pris ses premières photos dans les mines, que Wicky exposa pour la première fois son travail au Musée national d'art de La Paz, avant de le faire connaître dans toute la Bolivie, puis en Amérique du Sud, en Europe, au Mexique et aux États-Unis. Il rencontra partout un succès dont il ne s'enorgueillit jamais, continuant de se rendre plusieurs années encore à Potosi pour compléter et parfaire son œuvre et son hommage aux mineurs. L'ouvrage Mineros. Bolivia, qui était initialement censé marquer la fin de cette riche expérience, fut publié en 2002 dans une première édition en français, puis en 2007 dans une édition espagnole qui permit à Wicky de tenir la promesse faite aux mineurs de partager avec eux le résultat de ces longues années de travail, de confiance et parfois d'amitié :

J'ai acquis 600 exemplaires de l'édition espagnole que j'ai fait envoyer en Bolivie, et j'ai voyagé dans ces lieux qui ne figurent pas sur les cartes géographiques, pour offrir le livre aux mineurs que j'ai pu retrouver, mais surtout pour en remettre un exemplaire aux bibliothèques des écoles et collèges des centres miniers. [...] Après tant d'années partagées avec eux, il me semblait naturel de leur rendre, en partie, ce qu'ils m'avaient donné. (Ibid.)

26 Les premières réactions des mineurs, reconnaissants, émus ou fiers, bouleversèrent le photographe au point qu'il entreprit de consacrer un documentaire vidéo à ce retour dans les mines (ibid.), près de trente années après la naissance de son projet photographique. Accompagné d'une équipe sommaire et chargeant dans sa Jeep près de 600 exemplaires du livre Mineros. Bolivia, il parcourut les districts miniers dans lesquels il 
avait passé tant de temps, afin de filmer ses retrouvailles avec leurs habitants, quand ces derniers étaient encore en vie.

$\mathrm{Au}$ terme de près de quarante heures de tournage et de plusieurs mois consacrés au montage, le film Todos los días la noche "vit le jour" en 2010, et fut projeté à la Cinémathèque de La Paz, en présence de plusieurs dizaines de mineurs et d'autorités politiques qui saluèrent la démarche de Wicky et son engagement aux côtés des " travailleurs de l'ombre", trop souvent ignorés alors même qu'ils furent si fréquemment en première ligne des grands combats et des bouleversements de l'histoire nationale. D'une durée de presque une heure, au cours de laquelle le spectateur passe des profondeurs de la mine aux hauts sommets des Andes, du jour à la nuit et du sourire ému de certains mineurs aux larmes des autres, Todos los días la noche s'inscrit dans la droite lignée de Mineros. Bolivia, par son caractère sobre et respectueux et par la bienveillance dont il fait preuve à l'égard des hommes et femmes qu'il nous présente, sans artifices ni concessions. Les échanges avec les mineurs y alternent avec des explications sur l'histoire de l'exploitation des mines de la région et leur importance dans la vie politique et économique du pays, alors que le noir et blanc des clichés contraste avec l'éblouissante luminosité des prises de vue de l'Altiplano et les couleurs pastel de ses paysages spectaculaires.

Figure 14 : Canyon d'Antequera, Oruro.

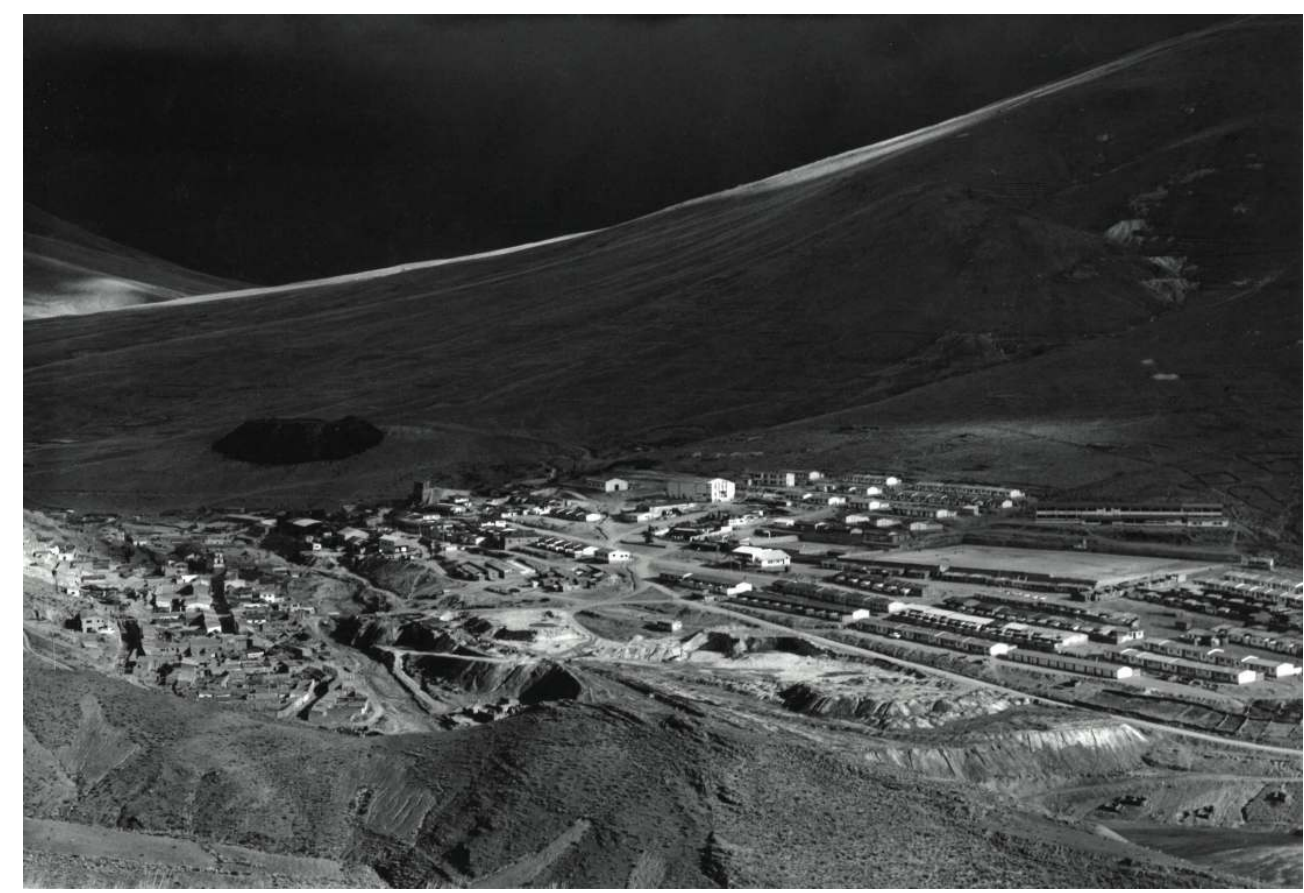

(c) Jean-Claude Wicky, 2002 (reproduit avec l'aimable autorisation de l'ayant-droit).

La rencontre avec ceux qui l'accueillirent au fil des années, l'émotion de certains d'entre eux et la joie des autres à la vue des photos sur lesquelles ils se reconnaissent avec surprise, ne sauraient cependant masquer ou amoindrir la dureté du documentaire de Jean-Claude Wicky, qui dénonce sobrement mais explicitement des conditions de vie indignes, sans jamais tomber dans le jugement, le prêche ou le pathos, mais sans non plus se mentir ni atténuer la réalité. Il révèle ainsi au spectateur le monde de la mine et ses visages avec justesse et pudeur, même si les témoignages poignants de certains des 
interviewés laissent souvent une sensation d'impuissance. À cet égard, le film de Wicky s'inscrit dans la continuité de son travail photographique, en ce qu'il donne la parole aux mineurs, après que les clichés leur ont (re)donné un visage et une visibilité dans le monde entier.

Les deux œuvres semblent ainsi dialoguer de façon presque atemporelle, tissant un lien non seulement entre les différentes étapes et formes de la démarche de Wicky, mais aussi entre deux époques distantes de près de quinze ans et pourtant si proches, que le film permet à son auteur de réunir, en parcourant à nouveau les mines dans lesquelles il avait pris ses clichés. Seule une dimension nouvelle, probablement due au format audiovisuel lui-même, imprègne ce que l'on pourrait considérer comme une sorte de deuxième volet de la rencontre de Wicky avec la mine et ses acteurs: la corde émotionnelle. En effet, outre l'impact de la vidéo, qui donne la parole à celles et ceux que la photo avait saisis sans pour autant jamais leur permettre de s'exprimer autrement que par le regard, l'artiste ne se rend plus dans les mines comme l'étranger qu'il était quinze ans auparavant : il n'est plus le «gringo » devant gagner la confiance des hommes et femmes qu'il souhaite photographier, mais bien une sorte de témoin ou de porte-parole, voire d'ami et parfois presque de " héros » pour certains mineurs dont il a changé la vie. C'est le cas de Félix Carmona, qui a du mal à retenir ses larmes en se revoyant âgé d'à peine quatorze ans, pris en photo par Wicky alors qu'il s'apprêtait à rejoindre la mine avec son père. Il raconte alors comment cette rencontre bouleversa son existence, entouré de ses trois filles et de son épouse que la caméra semble mettre mal à l'aise, à en juger par leur regard fuyant et leurs gestuelle incommodée :

À l'époque où cette photo a été prise, j'allais justement travailler à la mine avec mon père. J'étais obligé de travailler dans la mine, je ne pouvais pas étudier, j'aimais la mécanique mais je ne pouvais pas faire d'études, du fait de la situation économique de ma famille. C'est là que j'ai rencontré Jean-Claude alors que je partais travailler, et qu'il m'a demandé pourquoi je travaillais si jeune. J'ai répondu que la situation m'y obligeait car je devais aider ma famille, mes frères, et que la mine rapportait peu. C'est ainsi qu'a commencé cette histoire et que j'ai pu me consacrer à la mécanique ; c'est grâce à l'aide du Jean-Claude que je suis là où j'en suis aujourd'hui. (Ibid.)

Le mineur Valentín Mamani, avec plus de quarante ans d'activité dans la mine, reçoit pour sa part le livre des mains de Wicky avec une gratitude et une amitié empreintes d'une pudeur manifeste dans la façon qu'il a de tendre la main au photographe, qui s'empresse au contraire de le prendre dans ses bras: "Je suis très reconnaissant, réellement, c'est comme un trésor que je ramène chez moi pour ma famille, pour qu'ils sachent que, vraiment, tout ce temps où je les ai accompagnés n'a pas été vain. » (Ibid.) Ces éloges et témoignages d'amitié, qui constituent la principale différence avec le caractère sobre et brut des photographies, placent le documentaire sous un angle très différent qui laisse une part plus importante à la subjectivité et aux émotions, bien que toutes les scènes de retrouvailles ne soient pas nécessairement placées sous cet angle affectif.

31 D'autres donnent plus simplement la parole aux mineurs, Wicky disparaissant derrière la caméra et leur permettant de parler de leur quotidien et de leur réalité sans même évoquer son travail. Ce faisant, le film parle aussi en filigrane du rapport entre l'artiste et les sujets de ses photographies, dans une sorte de mise en abyme qui invite à oser regarder en face les méandres d'un monde qui pourrait nous sembler relever d'un passé révolu. C'est pourtant le contraire que nous disent la plupart des mineurs témoignant 
dans le documentaire, à l'instar d'Hilario Mamani dont les propos rappellent combien la mine reste d'une implacable dureté : «Notre travail est inhumain, mais ce serait pire de ne pas avoir de travail. » (Ibid.)

Figure 15 : Sans titre, Potosi.

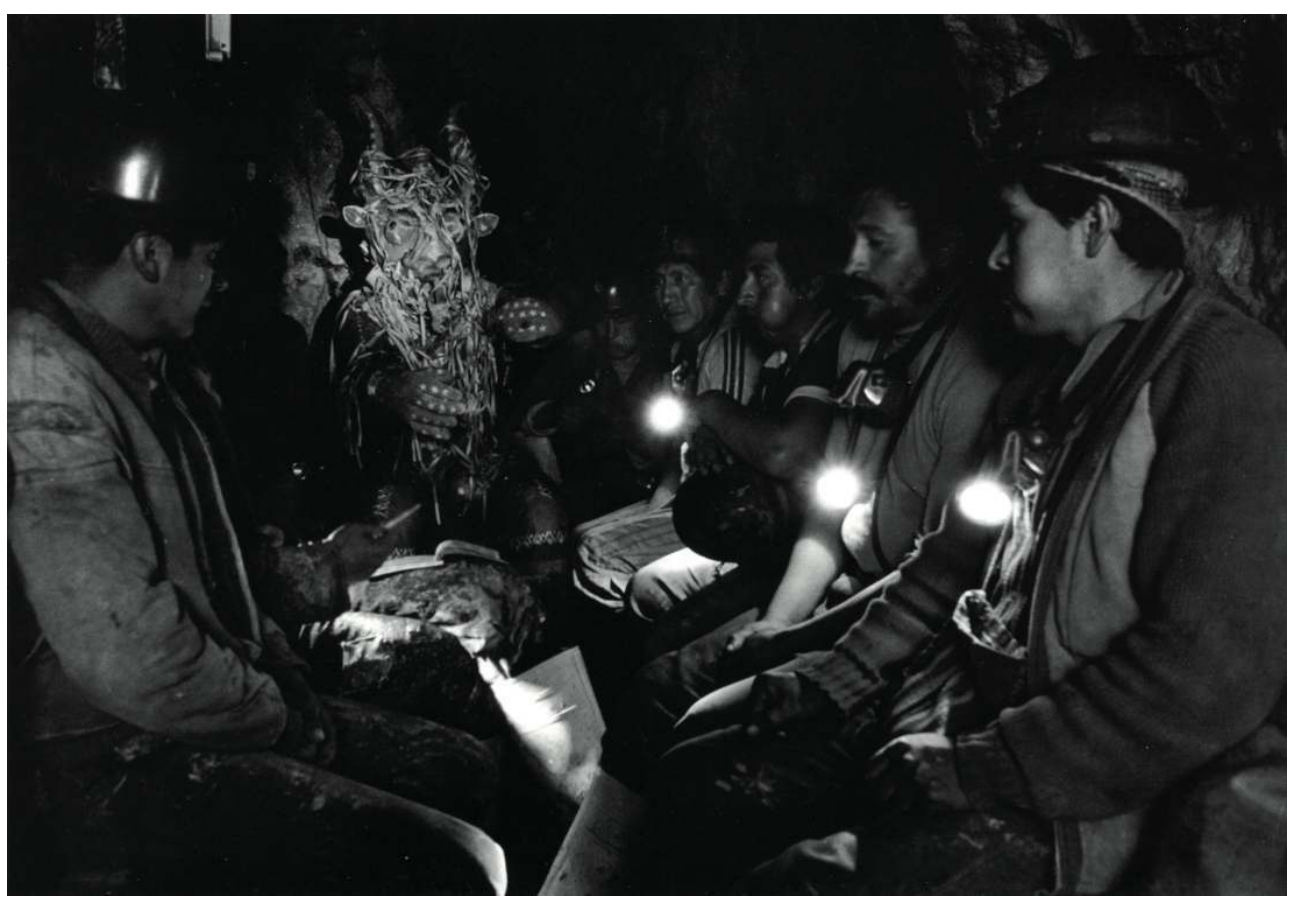

(c) Jean-Claude Wicky, 2002 (reproduit avec l'aimable autorisation de l'ayant-droit).

Un autre mineur, anonyme, illustre plus précisément cette affirmation, rappelant que ce travail ne connait comme rythme et règles que ceux que les mineurs s'imposent euxmêmes, dans un contexte économique qui les pousse à passer l'essentiel de leur temps dans la mine:«Les horaires de travail n'existent pas pour nous [...]. C'est le fonctionnement de notre travail : nous n'avons ni jours fériés ni week-end, si nous avons besoin de travailler et s'il est possible de le faire jour et nuit, nous restons plus longtemps ici [sous terre] que chez nous. » (Ibid.)

Et de poursuivre sur la principale motivation que lui et la plupart de ses collègues ont pour descendre dans la mine :

Tout parent pense à ses enfants et souhaite qu'ils puissent avoir un avenir meilleur; qu'ils puissent peut-être réussir ce que nous n'avons pas réussi, nous, qu'ils ne soient pas comme leurs parents. Leur donner ce qui est nécessaire pour qu'un jour ils deviennent quelqu'un, pas comme nous, qui ne sommes que des mineurs et la vie s'arrête là. (Ibid.)

La parole est aussi donnée aux palliris, d'abord en voix-off, sans visage particulier associé aux déclarations, comme si toutes parlaient d'une seule et même voix: "Qui peut se rappeler de nous, hein ? Personne.» (Ibid.) S'en suit le témoignage de l'une d'entre elles, Ana María Vaquera Mamani (quarante-six ans) n'ayant d'autre choix que de travailler pour nourrir sa famille: "Mon mari a eu un accident dans la mine, il est invalide, paralysé, il ne marchera plus jamais. C'est pourquoi je travaille aujourd'hui, pour ma famille, je suis le père et la mère, si je ne travaille pas, mes enfants ne mangent pas. » ( Ibid.) 

dose de luminosité en contrastant fortement avec les scènes souterraines, comme pour permettre au spectateur de reprendre son souffle ainsi que de la hauteur. La voix-off de Wicky est omniprésente, rythmant les images et les imprégnant de son accent français, qui à la fois fait sourire et resitue aussi l'œuvre et la démarche dans leur genèse : la volonté de témoignage d'un homme ayant posé un regard d'artiste étranger, puis rapidement de témoin engagé, sur un monde dont bien des Boliviens eux-mêmes ne soupçonnaient peut-être pas toujours la complexité. Le livre Mineros. Bolivia et le film Todos los días la noche permettent ainsi, chacun avec ses spécificités et limites, de porter un regard large et sensible sur le métier et la vie de personnes parfois déconsidérées ou un peu oubliées, que l'artiste nous révèle dans leur dimension humaine, leur dignité, leurs difficultés et leurs joies. Mais loin de se contenter de décrire, Jean-Claude Wicky cherche indéniablement à faire passer un message au public, comme s'il cherchait à sortir d'une forme d'anonymat séculaire les hommes, femmes et enfants qu'il a rencontrés dans la région de Potosi et à qui il souhaite rendre hommage.

Figure 16 : Vue de Potosi et du Cerro Rico.

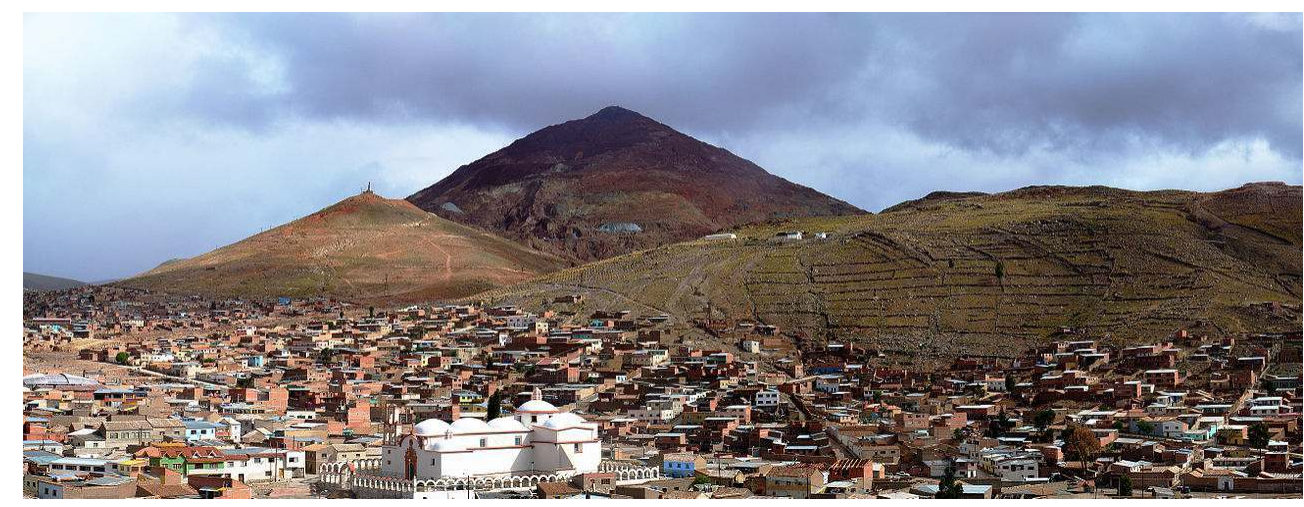

(C) Martin St-Amant, 2007.

\section{Photographes « humanistes »?}

Si l'image, animée ou figée, semble parfois bien dérisoire face à une réalité si éloignée de la nôtre, elle constitue pour Jean-Claude Wicky un engagement nécessaire avec le monde : «Lorsque l'on ne voit pas de changements, on a parfois l'impression que les photos ne servent à rien. Mais les photos sont à l'épreuve du temps et l'engagement de longue haleine ; c'est un pari nécessaire pour parler du monde et l'aimer.» (Ibid.) Un pari relevé par Wicky et par d'autres photographes ayant consacré une part importante de leur œuvre à la photographie documentaire, tels que Sebastião Salgado ou Willy Ronis ${ }^{13}$ à qui l'on doit la définition de l'école humaniste comme école du « regard du photographe qui aime l'être humain ».

37 C'est probablement dans le travail de Sebastião Salgado, photographe militant s'il en est, que l'on retrouve le plus de ressemblances avec celui de son contemporain Jean-Claude Wicky, tant du point de vue de leur démarche que dans la dimension esthétique. Dans un ouvrage tel que La main de l'homme: une archéologie de l'ère industrielle (Salgado, S., 1993), qui présente des clichés de travailleurs de vingt-six pays du monde, le recours exclusif à un noir et blanc puissant et expressif ainsi que la peinture à la fois crue et digne de 
l'humanité, font incontestablement penser aux photographies réunies dans Mineros. Bolivia ${ }^{14}$. Alliant un réalisme sans concession ni ethnocentrisme à la quête d'une représentation digne et noble de l'humanité face à des conditions de vie extrêmes, les œuvres de ces deux photographes visent à saluer le courage et le labeur des hommes et des femmes du monde, plus particulièrement des zones reculées ou meurtries de la planète. La photographie acquiert alors une dimension sociale, politique et engagée de premier ordre, devenant à la fois témoignage et dénonciation, hommage et invitation à la prise de conscience. Elle ne saurait cependant se suffire à elle-même, selon Sebastião Salgado: "Seules, mes photos ne peuvent rien. Il y a certainement des gens que mon travail a sensibilisés, mais de là à modifier la société... On peut participer au changement, mais en s'inscrivant dans un mouvement. » (Salgado, S., 2017)

C'est aussi dans leur volonté et leurs activités d'engagement que se retrouvent les deux photographes, devenus l'un et l'autre, chacun à leur manière, ambassadeurs de différentes luttes sociopolitiques en Bolivie et au Brésil. Alors que Salgado milite aux côtés de nombreuses associations (Reporters Sans Frontières, Unicef, OMS) et a fondé en 1998 l'ONG Instituto Terra pour reforester l'État du Minas Geiras ${ }^{15}$, Wicky milita aux côté des mineurs andins qu'il accompagna des décennies durant, afin que l'État bolivien et les coopératives privées améliorent leurs conditions de vie et de travail. Alors que Salgado a récemment intégré l'Académie des Beaux-arts (RFI, 2017), d'où il œuvre pour maintenir un lien actif entre la photographie et la sensibilisation à différentes causes de lutte, Wicky rencontra à plusieurs reprises les autorités nationales et scientifiques boliviennes, contribuant à faire connaître le quotidien des mineurs à des milliers de Boliviens des villes, souvent loin d'imaginer leur situation. Son amour de la Bolivie "minérale et ardente", de ses paysages à l'épreuve du temps et de ses gens aux regards profonds et expressifs, Jean-Claude Wicky les aura inlassablement partagés pendant plus de trente ans de son vivant, et ses photographies continueront de le faire de longues années encore. Et si la Suisse et le monde ont perdu un photographe le 31 juillet 2016, la Bolivie y a aussi en quelque sorte perdu un mineur de plus, revenu à jamais à la terre depuis laquelle il veille peut-être sur ses collègues aux côtés du Tío ; mais cela, seule « la montagne [le] sait, mais la montagne ne dit rien » (Wicky J.C., 2010), comme l'avait un jour déclaré la palliri doña Julia Villca à son ami Jean-Claude. 
Figure 17 : Intérieur de la mine.

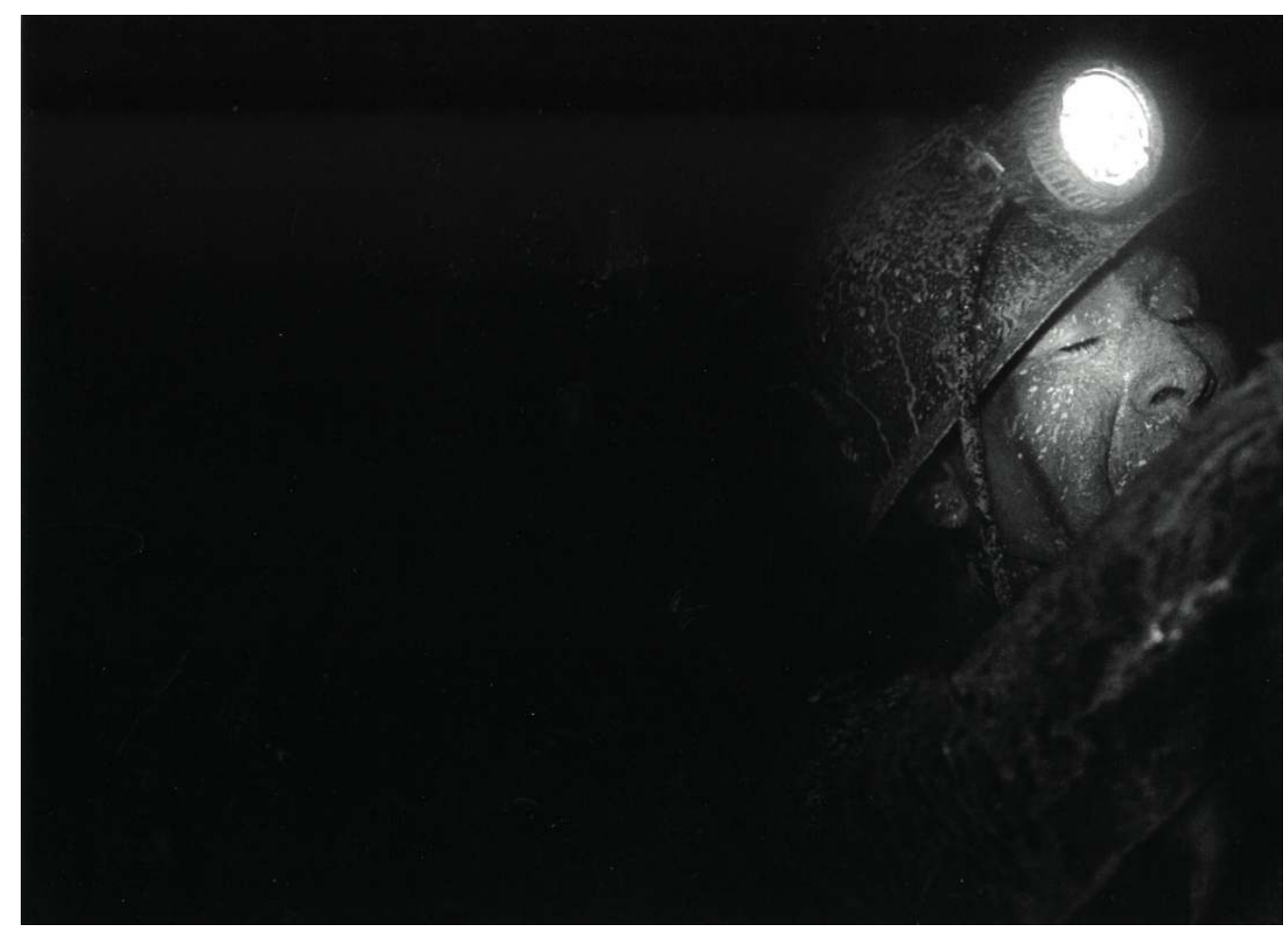

(c) Jean-Claude Wicky, 2002 (reproduit avec l'aimable autorisation de l'ayant-droit).

\section{BIBLIOGRAPHIE}

Absi, Pascale, Les ministres du diable. Le travail et ses représentations dans les mines de Potosi, Bolivie, Paris, L'Harmattan, 2003.

Arzáns de Orsúa y Vela, Bartolomé, Historia de la villa imperial de Potosí, 1676-1736, Hanke, Lewis et Mendoza Gunnar (éd.), Providence, Brown University Press, 1965 [1737].

Bernard, Jean-Pierre « La Bolivie et les compagnies productives de minerai d'étain », Revue française de science politique, vol. 2, n 17, avril 1967, p. 317-329.

Dunkerley, James, Orígenes del poder militar. Bolivia 1879-1935, 2e éd., La Paz, Plural Editores, 2003. Galeano, Eduardo, Memoria del fuego. III. El siglo del viento, México, Siglo XXI, 1986. Disponible en ligne : https://vivelatinoamerica.files.wordpress.com/2015/01/galeano_eduardomemoria_del_fuego_iii_el_siglo_del_viento.pdf.

Helmer, Marie, « Potosi à la fin du XVIII ${ }^{\mathrm{e}}$ siècle, 1776-1797 », Journal de la Société des Américanistes, tome 40,1951. p. 21-50.

Langue, Frédérique et Salazar-Soler, Carmen, Dictionnaire des termes miniers en usage en Amérique espagnole XVI ${ }^{e}-\mathrm{XIX} \mathrm{X}^{e}$ siècles, Paris, Éditions Recherche sur les Civilisations, 1993. 
Lavaud, Jean-Pierre, L'instabilité politique de l'Amérique latine : le cas de la Bolivie, Paris, Éditions de l'IHEAL, 1991. Disponbible en ligne : http://books.openedition.org/iheal/1030.

Le Gouill, Claude, « Imaginaires miniers et conflits sociaux en Bolivie : une approche multiniveaux du conflit de Mallku Khota », Cahiers des Amériques latines, n 82, 2016, p. 49-69.

Mesa, José, Gisbert, Teresa et Mesa Gisbert, Carlos, Historia de Bolivia, $7^{\mathrm{e}}$ éd., La Paz, Editorial Gisbert y Cía, 2008.

Poppe, René, Interior Mina, La Paz, Plural Editores, 2003 [1986].

Rico, Luis, « Mi amigo Jean-Claude Wicky », Página Siete, La Paz, 14 août 2016, http:// www.paginasiete.bo/letrasiete/2016/8/14/amigo-jean-claude-wicky-105791.html (consulté en avril 2018).

Salgado, Sebastião, La main de l'homme : une archéologie de l'ère industrielle, Paris, La Martinière, 1993.

Sanjinés, Jorge, et Grupo Ukamau, Teoría y práctica de un cine junto al pueblo, México, Siglo XXI, 1979.

Taboada Terán, Néstor, El precio del estaño, La Paz, Plural Editores, 2006 [1960].

Wicky, Jean-Claude, Todos los días la noche, film produit et distribué par l'auteur, 2010.

Mineros. Bolivia, Barcelone, Lunwerg Editores, 2007.

Mineros. Mineurs de Bolivie, Arles, Actes Sud, 2002.

Jean-Claude Wicky, Mineros, documentaire vidéo produit par le Museo de Etnografía y Folklore (MUSEF), La Paz, 1998. Disponible en ligne : https://www.youtube.com/watch ?v =PRCQQcVuqNg, consulté le 3 mars 2018.

"Jean-Claude Wicky », Zig Zag Café, entretien avec Jean-Philippe Rapp pour la Radio Télévision Suisse, 21 mars 2003, consultable en ligne sur : https://www.rts.ch/archives/tv/divers/zig-zagcafe/3479046-jean-claude-wicky.html (consulté le 3 avril 2018).

«Le photographe Sebastião Salgado s'installe à l'Académie des Beaux-Arts », Radio France Internationale, 6 décembre 2017 : http://www.rfi.fr/culture/20171206-sebastiao-salgadophotographe-s-installe-academie-beaux-arts (consulté le 4 juin 2018).

«Le photographe Willy Ronis est mort », Le Nouvel Obs, 13 septembre 2009 : https:// www.nouvelobs.com/culture/20090912.OBS0911/le-photographe-willy-ronis-est-mort.html (consulté le 4 juin 2018).

«Sebastião Salgado : “Seules, mes photographies ne peuvent rien" ", Télérama, 6 décembre 2017 : http://www.telerama.fr/sortir/sebastiao-salgado-seules,-mes-photos-ne-peuventrien,n5387206.php (consulté le 4 juin 2018).

« Tras cuatro siglos, Potosí supera el número de habitantes que tuvo en la colonia », La Razón, 17 mars 2016, http://www.la-razon.com/ciudades/Potosi-supera-siglos-habitantescolonia_0_2455554483.html (consulté en avril 2018).

\section{NOTES}

1. Le travail de Wicky a été exposé dans trente-six villes de douze pays, selon le site officiel du photographe. Plusieurs de ses clichés ont été acquis par des collections privées telles que : la Bibliothèque du Congrès de Washington, l'Institute of Art de Minneapolis, le Musée de l'Élysée de 
Lausanne, la Fondation suisse pour la photographie de Zurich ou encore le Centre historique minier Lewarde (France): https://www.touslesjourslanuit.com/es/accueil (consulté en mars 2018).

2. Le général Hugo Bánzer instaura une féroce dictature militaire entre 1971 et 1978, qui réprima rapidement l'opposition et les mouvements ouvriers, interdit les partis politiques, viola systématiquement les droits humains et s'associa aux États-Unis et aux dictatures anticommunistes de la région, coopérant dans le cadre de la Doctrine de Sécurité Nationale nordaméricaine et de l'opération Condor. La corruption et la dette du pays augmentèrent à une vitesse foudroyante, alors que s'ouvrait la grande époque du narcotrafic dans le pays. Il fut renversé en 1978 par son ancien ministre de l'intérieur, qui prit le pouvoir avant d'être renversé à son tour, trois mois plus tard. Sur l'histoire de la Bolivie et de la période militaire, on pourra consulter : Lavaud, Jean-Pierre (1991, chapitre II) ou Mesa, José, Gisbert, Teresa et Mesa Gisbert, Carlos (2008).

3. Dans Jean-Claude Wicky, Mineros, documentaire vidéo produit par le Museo de Etnografía y Folklore (MUSEF), La Paz, 1998 (consulté en mars 2018). Toutes les citations de cet article sont traduites de l'espagnol par l'auteur, sauf indication contraire.

4. Une ville alors bien plus peuplée que Londres ou Rome, qui comptaient respectivement environ 75000 et 45000 habitants en 1550. Sur l'histoire de la ville, voir Arzáns de Orsúa y Vela, Bartolomé (1965 [1737]). Pour un aperçu des mouvements démographiques de la ville, on pourra consulter « Tras cuatro siglos, Potosí supera el número de habitantes que tuvo en la colonia », La Razón, 17 mars 2016.

5. Il s'agissait des industriels et millionnaires Simón I. Patiño, Mauricio Hochschild et Carlos Víctor Aramayo, qui contrôlaient à eux trois près de $85 \%$ des ressources d'étain du pays et jouissaient à ce titre d'une immense influence sur les différents gouvernements au pouvoir entre les années 1920 et la nationalisation de leurs possessions à la suite de la Révolution nationale de 1952. Les barons de l'étain furent directement ou indirectement responsables de plusieurs massacres perpétrés dans les départements de Potosi et Oruro entre 1920 et 1950.

6. La date de 1923 est généralement considérée comme le point de départ du mouvement ouvrier minier bolivien et par la même occasion de ce funeste décompte, avec la "Masacre de Uncía " perpétrée par le gouvernement du président Bautista Saavedra pour protéger les intérêts du baron de l'étain Simón I. Patiño face aux grévistes.

7. Achevé en Europe au printemps 1971, quelques mois avant le coup d'État militaire d'Hugo Bánzer, le film El coraje del pueblo relate l'épisode du "massacre de la San Juan », survenu le 24 juin 1967 à Catavi durant le gouvernement du général Barrientos. Le film fut instantanément interdit en Bolivie, et Sanjinés ne put rentrer dans son pays qu'à la chute du dictateur, après sept années d'exil durant lesquelles il continua cependant de réaliser des films, au Pérou puis en Équateur. Sur la dimension politique et engagée du cinéma de Sanjinés, voir : Sanjinés Jorge et Grupo Ukamau (1979).

8. On retrouve par exemple une lampe à carbure, un marteau pneumatique, de la dynamite, des feuilles de coca.

9. Le «Tío»- littéralement «l'oncle »-, aussi connu sous le nom de Supay, est le patron des mines et le maître de l'«inframundo » dans lequel pénètrent les mineurs pour exploiter ses richesses. Ces derniers doivent à ce titre se mettre sous sa protection en le rassasiant par des offrandes de feuille de coca, d'eau-de-vie, de cigarettes et de serpentins, témoignages de la déférence dont ils doivent faire preuve pour prétendre sortir indemnes de son domaine. Figure tutélaire du monde sous-terrain et divinité plus ambivalente que néfaste, le Tío est capable de se montrer impitoyable et meurtrier autant que de combler ceux qu'il prend sous son aile en leur révélant de riches filons de minerai. C'est seulement à l'époque coloniale qu'il fut associé au Diable, probablement du fait de leur dimension chtonienne commune et de la menace qu'ils 
présentent pour quiconque s'aventure dans leur royaume. Pour une étude approfondie de la relation entre les mineurs et le Tío, on pourra consulter Absi, Pascale (2003).

10. René Poppe est aussi connu pour ses nombreux contes sur la mine : El paraje del tío y otros relatos mineros, La Paz, Ediciones Piedra Libre, 1979 ; Cuentos Mineros, La Paz, ISLA, 1985.

11. Le grand romancier bolivien Néstor Taboada Terán écrivait à ce propos, dans son roman historique El precio del estaño sur la révolte des mineurs de Catavi en 1942, que « las mujeres hacen desaparecer las vetas con su presencia », Taboada Terán, Néstor (2006 [1960] : 66).

12. Le terme "palliri» vient de l'aymara «palla-ri » qui signifie littéralement «celui/celle qui ramasse/collecte». Pour une meilleure compréhension des termes spécifiques au monde de la mine, on pourra consulter : Langue, Frédérique et Salazar-Soler, Carmen (1993).

13. On réduit souvent le travail de Ronis à ses photographies sur le Paris pittoresque des quartiers populaires de Belleville ou Ménilmontant, oubliant que nombre de ses clichés furent pris aux côtés des ouvriers, dont il partageait les luttes sociales et politiques de l'époque (grèves et manifestations, syndicalisme, etc.). Son travail documentaire pour la SNCF, sur le retour des prisonniers de guerre, s'inscrit aussi dans cette veine de photographie documentaire « humaniste ». Voir : « Le photographe Willy Ronis est mort », Le Nouvel Obs, 13 septembre 2009.

14. De plus, le passage du cliché photographique au format cinématographique constitue un autre rapport entre les deux artistes, puisque le travail de Salgado a été porté au grand écran par le film Le Sel de la Terre, coréalisé en 2014 par le cinéaste Wim Wenders et le scénariste Juliano Ribeiro Salgado, fils du photographe.

15. Après des années de travail et plusieurs millions d'arbres plantés, les Salgado travaillent dorénavant sur un projet de récupération et de sauvegarde d'un affluent du fleuve Rio Doce, dévasté par la coulée de boue toxique du 5 novembre 2015, suite à la rupture de deux bassins de rétention de la compagnie minière Samarco.

\section{RÉSUMÉS}

"Je suis entré dans le pays par sa porte la plus douloureuse », déclara le photographe suisse JeanClaude Wicky au terme d'un long et patient travail de photographie documentaire sur les mines boliviennes de Potosi, réalisé entre 1984 et 2001 dans cet univers très codifié, hostile et néanmoins indissociable de l'histoire de la Bolivie. Cet article se propose de présenter l'ouvrage Mineros. Bolivia (2007), fruit de ce travail salué par la critique et le public pour sa qualité artistique et documentaire. Plus qu'une réflexion sur la dimension anthropologique et historique de l'œuvre de Wicky, dont on évoquera cependant certains points permettant de mieux appréhender les photographies, c'est à une analyse de la démarche de l'artiste que l'on s'intéressera plus particulièrement ici, en étudiant certaines caractéristiques des clichés, qui introduisent le spectateur dans un univers confiné, brut et âpre, sans pour autant jamais chercher à déformer ou caricaturer la réalité complexe dont ils témoignent. On se penchera aussi sur une autre étape de la démarche de Wicky, qui décida, après avoir exposé ses photographies dans plusieurs grandes villes aux quatre coins du monde ${ }^{1}$, de remettre à chacun des mineurs immortalisés par ses clichés un exemplaire du livre, une fois celui-ci édité en version espagnole (2007). Les réactions émues de ces derniers le décidèrent à consacrer un documentaire vidéo à ce retour dans les mines, vingt-six ans après le début du projet initial. Il réalisa ainsi le film documentaire Todos los días la noche (2010), dans lequel alternent les scènes de rencontre avec les mineurs et les explications sur l'histoire de la région, tout en introduisant une subtile réflexion 
sur la relation de l'artiste avec les personnes qui l'ont inspiré et ont nourri son travail pendant des décennies.

«Ingresé al país por su puerta más dolorosa », declaró el fotógrafo suizo Jean-Claude Wicky al concluir un largo y paciente trabajo de fotografía documental sobre las minas bolivianas de Potosí, realizado entre 1984 y 2001 en aquel universo complejo y hostil a la vez que indisociable de la historia de Bolivia. Este artículo se propone presentar la obra Mineros. Bolivia (2007), resultado de su trabajo cuya calidad artística y documental fue alabada por la crítica y el público. Más que una reflexión sobre la dimensión antropológica e histórica de dicha obra, se tratará aquí de analizar y comentar algunas características de las fotografías, que introducen al espectador en un universo confinado, bruto y duro, sin deformar ni caricaturizar nunca la compleja realidad que pintan. También nos interesaremos por la iniciativa de Wicky quien decidió, después de exponer su trabajo en varias ciudades del mundo, entregar un ejemplar del libro a cada uno de los mineros plasmados en sus fotografías. La gratitud y la emoción de aquéllos lo animaron a dedicar un video documental a este regreso a la mina, veintiséis años después del proyecto inicial. Realizó entonces el filme Todos los días la noche (2010), en el que se mezclan los encuentros con los mineros y las explicaciones sobre la historia minera de la región, al mismo tiempo que propone una sutil reflexión sobre la relación que une el artista a las personas que lo inspiraron y alimentaron su trabajo durante décadas.

"I entered the country by its most painful door" declared the Swiss photographer Jean-Claude Wicky at the end of a long and patient work of documentary photography about the Bolivian mines of Potosi, realized between 1984 and 2001 in a hostile and fierce universe, inseparable from the history of Bolivia. This article proposes to present the book Mineros. Bolivia (2007), result of this work hailed by critics for its artistic and documentary quality. More than a reflection on the anthropological and historical dimension of the work, the point of this article is to study some of the characteristics of the pictures, which introduce the public into a confined, raw and bitter world, without ever distorting or caricaturing the complex reality of which it testifies. We will also take an interest in the process by which Wicky decided, after exposing his work in many cities in the world, to give each of the miners immortalized by his photographs a copy of the book. Their gratefulness and emotional reactions decided him to dedicate a video documentary to this return to the mines, twenty-six years after the beginning of the project. He directed the documentary film Todos los días la noche (2010), in which scenes of encounter with mineworkers alternate with explanations of the mining history of the region, while introducing a subtle reflection on the relationship between the artist and the people who inspired him and fed his work for decades.

\section{INDEX}

Mots-clés : Bolivie, Potosi, mines, Jean-Claude Wicky, photographie documentaire

Palabras claves : Bolivia, Potosí, minas, Jean-Claude Wicky, fotografía documental

Keywords : Bolivia, Potosi, mines, Jean-Claude Wicky, documentary photography

\section{AUTEUR}

\section{BAPTISTE LAVAT}

Maître de conférences en civilisation hispano-américaine à l'Université Paris 12 (Université Paris-Est-Créteil-Val de Marne - laboratoire IMAGER). Spécialiste du carnaval d'Oruro et des 
festivités boliviennes, il travaille principalement sur les notions d'identité, de pouvoir, de mémoire et de sociabilité dans les pratiques populaires andines (culturelles, religieuses, rituelles), ainsi que dans les arts (cinéma, musique, photographie), en Bolivie et dans différents pays latino-américains. lavat.baptiste@gmail.com 